DAMTP-2006-9

\title{
Anti-self-dual conformal structures with null Killing vectors from projective structures
}

\author{
Maciej Dunajski* and Simon West ${ }^{\dagger}$ \\ Department of Applied Mathematics and Theoretical Physics \\ University of Cambridge \\ Wilberforce Road, Cambridge CB3 OWA, UK
}

January 17,2006

Dedicated to the memory of Jerzy Plebański

\begin{abstract}
Using twistor methods, we explicitly construct all local forms of fourdimensional real analytic neutral signature anti-self-dual conformal structures $(M,[g])$ with a null conformal Killing vector. We show that $M$ is foliated by anti-self-dual null surfaces, and the two-dimensional leaf space inherits a natural projective structure. The twistor space of this projective structure is the quotient of the twistor space of $(M,[g])$ by the group action induced by the conformal Killing vector.

We obtain a local classification which branches according to whether or not the conformal Killing vector is hyper-surface orthogonal in $(M,[g])$. We give examples of conformal classes which contain Ricci-flat metrics on compact complex surfaces and discuss other conformal classes with no Ricci-flat metrics.
\end{abstract}

*email M.Dunajski@damtp.cam.ac.uk

$\dagger$ †email S.D.C.West@damtp.cam.ac.uk 


\section{Introduction}

The anti-self-duality (ASD) condition in four dimensions seems to underlie the concept of integrability of ordinary and partial differential equations [29]. Many lower dimensional integrable models (KdV, NlS, Sine-Gordon, ...) arise as symmetry reductions of the ASD Yang-Mills equations on a flat background, and various solution generation techniques are reductions of the twistor correspondence [19]. Other integrable models (dispersionless Kadomtsev-Petviashvili, $S U(\infty)$ Toda, ...) are reductions of the ASD conformal equations which say that the self-dual Weyl tensor of a conformal class of metrics vanishes [30, 7. Generalisations to ASD Yang-Mills on ASD conformal background are also possible [27, 4].

In all cases the main interest is in conformal structures of signature ++-- which are called neutral, as the reductions can lead to interesting hyperbolic and parabolic equations. There are no non-trivial ASD structures in the Lorentzian signature ++ +- , and the reductions from Riemannian manifolds can only yield elliptic equations thus ruling out interesting soliton dynamics.

The main gap in the programme to classify the reductions of ASD neutral conformal structures was understanding the reductions by a null conformal Killing vector. We embarked on this project hoping to incorporate more integrable systems into the framework of of anti-self-duality, but we have found (Theorem 2) that the resulting geometry is a completely solvable system.

Let $(M,[g])$ be a four dimensional real analytic neutral ASD conformal structure. We say that $K$ is a null conformal Killing vector if it satisfies

$$
\mathfrak{L}_{K} g=\eta g, \quad g(K, K)=0,
$$

for some $g \in[g]$, where $\eta$ is a function on $M$, and $\mathfrak{L}$ is the Lie-derivative.

When studying conformal structures with non-null conformal Killing vectors, it is natural to look at the space of Killing vector trajectories, since this will inherit a non-degenerate conformal structure. In the case of a null conformal Killing vector, the situation is different. The space of trajectories inherits a degenerate conformal structure. We find that it is necessary to go down one dimension more, and consider a two dimensional space $U$ of anti-self-dual totally null surfaces in $M$, called $\beta$-surfaces, containing $K$, which exist as a consequence of the conformal Killing equation. It turns out that there is a naturally defined projective structure $[\Gamma]$ on $U$. Moreover, we show that the twistor spaces of $(M,[g])$ and $(U,[\Gamma])$ are related by dimensional reduction. Specifically, the twistor space $Z$ of $(U,[\Gamma])$ is the space of trajectories of a vector field on the twistor space $\mathcal{P} \mathcal{T}$ of $(M,[g])$ corresponding to $K$. Projective structures are just equivalence classes of torsion-free connections, which do not need to satisfy any equations; this underlies the complete solvability of null reductions, and contrasts 
with the non-null case where one obtains Einstein-Weyl structures [13], and associated integrable systems 30, 7, 6, 4,

In Section 2 we derive some elementary properties of null conformal Killing vectors. Section 3 is an introduction to projective structures. In Section 4 we prove the following:

Theorem 1. Let $(M,[g])$ be a four dimensional real analytic neutral ASD conformal structure with a null conformal Killing vector $K$. Let $U$ be the two dimensional space of $\beta$-surfaces containing $K$. Then there is a naturally defined projective structure on $U$, whose twistor space is the space of trajectories of a distribution $\widehat{\mathcal{K}}$ induced on $\mathcal{P} \mathcal{T}$ by the action of $K$ on $M$.

In Section 5 we investigate the local form of ASD conformal structures with null Killing vectors. This is expressed in the following theorem:

Theorem 2. Let $(M,[g], K)$ be a smooth neutral signature ASD conformal structure with null conformal Killing vector. Then there exist local coordinates $(t, x, y, z)$ and $g \in[g]$ such that $K=\partial_{t}$ and $g$ has one of the following two forms, according to whether the twist $\mathbb{K} \wedge d \mathbb{K}$ vanishes or not $(\mathbb{K}:=g(K,)$.$) :$

1. $\mathbb{K} \wedge d \mathbb{K}=0$.

$$
\begin{aligned}
g= & \left(d t+\left(z A_{3}-Q\right) d y\right)(d y-\beta d x)- \\
& \left(d z-\left(z\left(-\beta_{y}+A_{1}+\beta A_{2}+\beta^{2} A_{3}\right)\right) d x-\left(z\left(A_{2}+2 \beta A_{3}\right)+P\right) d y\right) d x,
\end{aligned}
$$

where $A_{1}, A_{2}, A_{3}, \beta, Q, P$ are arbitrary functions of $(x, y)$.

2. $\mathbb{K} \wedge d \mathbb{K} \neq 0$.

$$
\begin{array}{r}
g=\left(d t+A_{3} \partial_{z} G d y+\left(A_{2} \partial_{z} G+2 A_{3}\left(z \partial_{z} G-G\right)-\partial_{z} \partial_{y} G\right) d x\right)(d y-z d x) \\
-\partial_{z}^{2} G d x\left(d z-\left(A_{0}+z A_{1}+z^{2} A_{2}+z^{3} A_{3}\right) d x\right),
\end{array}
$$

where $A_{0}, A_{1}, A_{2}, A_{3}$ are arbitrary functions of $(x, y)$, and $G$ is a function of $(x, y, z)$ satisfying the following PDE:

$$
\left(\partial_{x}+z \partial_{y}+\left(A_{0}+z A_{1}+z^{2} A_{2}+z^{3} A_{3}\right) \partial_{z}\right) \partial_{z}^{2} G=0 .
$$

The functions $A_{\alpha}(x, y)$ in the metrics (1.2) and (1.3) determine projective structures on the two dimensional space $U$ in the following way. A general projective structure corresponds to a second-order ODE

$$
\frac{d^{2} y}{d x^{2}}=A_{3}(x, y)\left(\frac{d y}{d x}\right)^{3}+A_{2}(x, y)\left(\frac{d y}{d x}\right)^{2}+A_{1}(x, y)\left(\frac{d y}{d x}\right)+A_{0}(x, y) .
$$


In (1.3) all the $A_{\alpha}, \alpha=0,1,2,3$ functions occur explicitly in the metric. In (1.2) the function $A_{0}$ does not explicitly occur. It is determined by the following equation:

$$
A_{0}=\beta_{x}+\beta \beta_{y}-\beta A_{1}-\beta^{2} A_{2}-\beta^{3} A_{3},
$$

as is shown in the proof of the theorem. If one interprets $z$ as a fibre coordinate on the projective tangent bundle of the $(x, y)$ space, then (1.4) says that $\partial_{z}^{2} G$ is constant along the projective structure spray, (compare formula 3.4).

Note that in both cases the Killing vector is $\partial_{t}$ and is pure Killing (this comes from choosing a suitable $g \in[g]$ ). The non-twisting case (1.2) is a natural conformal generalisation of Ricci-flat pp waves. The twisting case (1.3) is a neutral analog of the Fefferman conformal class [10]. As special cases of (1.3) we recover some examples of [20], where neutral metrics were related to second order ODEs.

The aim of Section [6 is to put our results into a broader context. We examine some examples found by different means in the light of our results. We find necessary and sufficient conditions on the underlying projective structure in order for there to exist (pseudo) hyper-complex metrics with triholomorphic $K$ within a conformal class. A special case of the metric (1.2) yields a compact example of a Ricci-flat metric on a Kodaira surface of a special type.

We consider how to construct conformal structure twistor spaces from projective structure twistor spaces in Section[7. The more involved spinor calculations are moved to the Appendix.

\section{Null Conformal Killing Vectors}

\subsection{Spinors in neutral signature}

We will denote by $(M,[g])$ a local patch of $\mathbb{R}^{4}$ endowed with a neutral signature conformal structure $[g]$. That is, $[g]$ is an equivalence class of neutral signature metrics with the equivalence relation $g \sim e^{c} g$ for a some function $c$ on $M$.

Any neutral metric $g$ on $M$ can be put in the following form:

$$
g=2\left(\theta^{00^{\prime}} \odot \theta^{11^{\prime}}-\theta^{01^{\prime}} \odot \theta^{10^{\prime}}\right)=\epsilon_{A B} \epsilon_{A^{\prime} B^{\prime}} \theta^{A A^{\prime}} \otimes \theta^{B B^{\prime}},
$$

where $\epsilon_{A B}, \epsilon_{A^{\prime} B^{\prime}}$ are antisymmetric matrices with $\epsilon_{01}=\epsilon_{0^{\prime} 1^{\prime}}=1$. The four (real) basis one-forms $\theta^{A A^{\prime}}$ for $A=0,1, A^{\prime}=0,1$ are called a tetrad. The algebraic dual vector basis is denoted $\mathbf{e}_{A A^{\prime}}$, and is defined by $\theta^{A A^{\prime}}\left(\mathbf{e}_{B B^{\prime}}\right)=\delta_{B}^{A} \delta_{B^{\prime}}^{A^{\prime}}$. Any vector $V$ at a point can be written $V^{A A^{\prime}} \mathbf{e}_{A A^{\prime}}$, and this exhibits an isomorpism

$$
T M \cong S \otimes S^{\prime}
$$


where $S, S^{\prime}$ are two-dimensional real vector bundles known as the unprimed spin bundle and the primed spin bundle respectively. For a general manifold $M$ there is a topological obstruction to (2.2) but we are working locally so it always holds.

Using a particular choice of tetrad, a section $\mu$ of $S$ is denoted $\mu^{A}, A=0,1$. Similarly $\nu_{A}$ is a section of $S^{*}, \kappa^{A^{\prime}}$ a section of $S^{\prime}$ and $\tau_{A^{\prime}}$ a section of $S^{\prime *}$, where * denotes the dual of a bundle. The natural pairing $S \times S^{*} \rightarrow \mathbb{R}$ is given by $\mu^{A} \nu_{A}$, using the summation convention, and similarly for primed spinors. We sometimes use the notation $\mu^{A^{\prime}} \nu_{A^{\prime}}=\mu . \nu$. This product is not commutative, we have $\mu . \nu=-\nu . \mu$.

It follows from (2.1) that $g(V, V)=\operatorname{det} V^{A A^{\prime}}$. If $V$ is null, then this gives $V^{A A^{\prime}}=$ $\mu^{A} \kappa^{A^{\prime}}$. Abstractly, if $V$ is null then $V=\mu \otimes \kappa$ under the isomorphism (2.2), where $\mu$ and $\kappa$ are sections of $S$ and $S^{\prime}$ respectively.

The relation (2.1) can be written abstractly as

$$
g=\epsilon \otimes \epsilon^{\prime}
$$

under the isomorphism (2.2). $\epsilon$ and $\epsilon^{\prime}$ are symplectic structures on $S$ and $S^{\prime}$. These give isomorphisms $S \cong S^{*}$ and $S^{\prime} \cong S^{*}$ by $\mu \rightarrow \epsilon(\mu,$.$) , for \mu$ a section of $S$, and similarly for $S^{\prime}$. Given a choice of tetrad, the spinors $\epsilon$ and $\epsilon^{\prime}$ are written $\epsilon_{A B}$ and $\epsilon_{A^{\prime} B^{\prime}}$, where we drop the prime on the latter because no confusion can arise due to the indices. Note these are anti-symmetric in $A B$ and $A^{\prime} B^{\prime}$. Then the isomorphism $S \cong S^{*}$ is given in the trivialization by $\mu^{A} \rightarrow \mu^{B} \epsilon_{B A}:=\mu_{A}$ and similarly for primed spinors.

There are useful isomorphisms

$$
\Lambda_{+}^{2} \cong \operatorname{Sym}\left(S^{*} \otimes S^{*}\right), \quad \Lambda_{-}^{2} \cong \operatorname{Sym}\left(S^{*} \otimes S^{*}\right),
$$

where $\Lambda_{+}, \Lambda_{-}$are the bundles of self-dual and anti-self-dual two-forms, using an appropriate choice of volume form for the Hodge-* operator. In the local trivialization, the isomorphisms (2.3) are expressed by the following formula for a two-form $F$ in spinors:

$$
F_{a b}=F_{A A^{\prime} B B^{\prime}}=\phi_{A^{\prime} B^{\prime}} \epsilon_{A B}+\psi_{A B} \epsilon_{A^{\prime} B^{\prime}},
$$

where $\phi_{A^{\prime} B^{\prime}}, \psi_{A B}$ are symmetric. The $\phi_{A^{\prime} B^{\prime}}$ term is the self-dual component of $F$ and the $\psi_{A B}$ is the anti-self-dual component.

The vector bundles $S, S^{\prime}$ and their duals inherit connections from the Levi-Civita connection of $T M$ (see Appendix A). These are the unique torsion-free connections defined so that the sections $\epsilon$ and $\epsilon^{\prime}$ are covariantly constant. Then covariant differentiation on either side of (2.2) is consistent.

A primed spinor $\kappa^{A^{\prime}}$ at a point corresponds to a totally null self-dual two-plane spanned by $\kappa^{A^{\prime}} \mathbf{e}_{A A^{\prime}}, A=1,2$, whilst an unprimed spinor corresponds to an antiself-dual two-plane in a similar way. In twistor theory, these two-planes are called $\alpha$-planes and $\beta$-planes respectively. 


\subsection{Null conformal Killing vectors in neutral signature}

Suppose $g$ is a neutral metric with a conformal Killing vector $K$. Then $\mathfrak{L}_{K}\left(e^{c} g\right)=$ $\left(K\left(e^{c}\right)+e^{c} \eta\right) g$, so $K$ is a conformal Killing vector for the conformally rescaled metric, and we can refer to $K$ as a conformal Killing vector for the conformal structure $[g]$.

Now suppose $g$ has a null conformal Killing vector $K$. We shall show (Lemma 11) that $M$ is foliated in two different ways, by self-dual and anti-self-dual surfaces, whose leaves intersect tangent to $K$. This is a property of the conformal structure $[g]$, since the Hodge- $*$ acting on 2 -forms is conformally invariant.

The spinor form of the conformal Killing equation is:

$$
\nabla_{a} K_{b}=\phi_{A^{\prime} B^{\prime}} \epsilon_{A B}+\psi_{A B} \epsilon_{A^{\prime} B^{\prime}}+\frac{1}{2} \eta \epsilon_{A B} \epsilon_{A^{\prime} B^{\prime}}
$$

where $\phi_{A^{\prime} B^{\prime}}, \psi_{A B}$ are the self-dual and anti-self dual parts of the 2 -form $\nabla_{[a} K_{b]}$, and $\eta$ is a function on $M$.

Since $K$ is null, we have $K=\iota \otimes o$, where $\iota$ is a section of $S$ and $o$ a section of $S^{\prime}$. Choosing a null tetrad, and a trivialization of $S$ and $S^{\prime}$, we have $K^{A A^{\prime}}=\iota^{A} O^{A^{\prime}}$. These spinors are defined up to multiplication by a non-zero function $\alpha$, since $K^{A A^{\prime}}=$ $\iota^{A} o^{A^{\prime}}=\left(\alpha \iota^{A}\right)\left(o^{A^{\prime}} / \alpha\right)$.

Lemma 1. Let $K=\iota^{A} O^{A^{\prime}} \boldsymbol{e}_{A A^{\prime}}$ be a null conformal Killing vector. Then

1. The following algebraic identities hold:

$$
\begin{aligned}
\iota^{A} \iota^{B} \psi_{A B} & =0, \\
O^{A^{\prime}} o^{B^{\prime}} \phi_{A^{\prime} B^{\prime}} & =0 .
\end{aligned}
$$

2. $\iota^{A}$ and $o^{A^{\prime}}$ satisfy

$$
\begin{aligned}
\iota^{A} \iota^{B} \nabla_{B B^{\prime}} \iota_{A} & =0, \\
o^{A^{\prime}} o^{B^{\prime}} \nabla_{B B^{\prime}} O_{A^{\prime}} & =0 .
\end{aligned}
$$

Remark. The equations (2.7), (2.8) are equivalent to the statement that the distributions spanned by $\iota^{A} \mathbf{e}_{A A^{\prime}}$ and $o^{A^{\prime}} \mathbf{e}_{A A^{\prime}}$ are Frobenius integrable (see Appendix). Equations of this type are often called 'geodesic shear free' equations, since in the Lorentzian case they result in shear-free congruences of null geodesics.

Proof. Using $K_{A A^{\prime}}=\iota_{A} O_{A^{\prime}}$, the Killing equation (2.4) becomes

$$
o_{A^{\prime}} \nabla_{B B^{\prime}} \iota_{A}+\iota_{A} \nabla_{B B^{\prime}} o_{A^{\prime}}=\phi_{A^{\prime} B^{\prime}} \epsilon_{A B}+\psi_{A B} \epsilon_{A^{\prime} B^{\prime}}+\frac{1}{2} \eta \epsilon_{A B} \epsilon_{A^{\prime} B^{\prime}}
$$


Contracting both sides with $\iota^{A} O^{A^{\prime}}$ gives

$$
0=o^{A^{\prime}} \iota_{B} \phi_{A^{\prime} B^{\prime}}+\iota^{A} o_{B^{\prime}} \psi_{A B}+\frac{1}{2} \eta \iota_{B} O_{B^{\prime}}
$$

Multiplying by $\iota^{B}$ and $o^{B^{\prime}}$ respectively leads to (2.5) and (2.6). To get (2.7) and (2.8) multiply (2.9) by $\iota^{A} \iota^{B}$ and $o^{A^{\prime}} O^{B^{\prime}}$.

We have found that $M$ is foliated in two different ways by totally null surfaces. Those determined by $O^{A^{\prime}}$ are self-dual and are called $\alpha$-surfaces, and those determined by $\iota^{A}$ are anti-self-dual and are called $\beta$-surfaces. It is clear that the $\alpha$-surfaces and $\beta$-surfaces of Lemma 1 intersect on integral curves of $K$. Denote the $\beta$-surface distribution by $\mathcal{D}_{\beta}$; this will be used later.

It is appropriate here to recall the Petrov-Penrose classification [22] of the algebraic type of a Weyl tensor. In split signature this applies separately to $C_{A B C D}$ and $C_{A^{\prime} B^{\prime} C^{\prime} D^{\prime}}$. In our case $C_{A^{\prime} B^{\prime} C^{\prime} D^{\prime}}=0$ and we are concerned with the algebraic type of $C_{A B C D}$. When we refer to the algebraic type we will be referring to the algebraic type of $C_{A B C D}$. One can form a real polynomial of fourth order $P(x)$ by defining $\mu^{A}=(1, x)$ and setting $P(x)=\mu^{A} \mu^{B} \mu^{C} \mu^{D} C_{A B C D}$. The Petrov-Penrose classification refers to the position of roots of this polynomial, for example if there are four repeated roots then we say $C_{A B C D}$ is type $\mathrm{N}$. If there is a repeated root the metric is called algebraically special. There are additional complications in the split signature case [16] arising from the fact that real polynomials may not have real roots.

The split signature version of the Goldberg-Sachs theorem together with (2.7) implies that any Ricci-flat ASD space with null conformal Killing vector is algebraically special. In fact the vacuum condition can be removed if $K$ is non-twisting; we will discuss this further in Section 6.5.

It also follows from the Killing equations and the fact that $K$ is null that

$$
K^{b} \nabla_{b} K_{a}=\frac{1}{2} \eta K_{a}
$$

Thus $K$ is automatically geodesic, and if it is pure then its trajectories are parameterized by an affine parameter.

\section{$3 \quad$ Projective structures}

Let $(U,[\Gamma])$ be a local two dimensional real projective structure. That is, $U$ is a local patch of $\mathbb{R}^{2}$, and $[\Gamma]$ is an equivalence class of torsion-free connections whose unparameterized geodesics are the same. Then in a local trivialization, equivalent torsion-free connections are related in the following way:

$$
\tilde{\Gamma}_{j k}^{i}-\Gamma_{j k}^{i}=a_{j} \delta_{k}^{i}+a_{k} \delta_{j}^{i},
$$


for functions $a_{i}$ on $U$, and $i, j, k=1,2$. Note that this is a tensor equation since the difference between two connections is a tensor. The $a_{i}$ on the RHS are the components of a one-form.

The geodesics satisfy the following ODE:

$$
\frac{d^{2} s^{i}}{d t^{2}}+\Gamma_{j k}^{i} \frac{d s^{j}}{d t} \frac{d s^{k}}{d t}=v \frac{d s^{i}}{d t}
$$

where $s^{i}$ are local coordinates of $U$, and $t$ is a parameter, which is called affine if $v=0$.

One can associate a second-order ODE to a projective structure by picking a connection in the equivalence class, choosing local coordinates $s^{i}=(x, y)$ say, and eliminating the parameter from the geodesic equations. The resulting equation determines the geodesics in terms of the local coordinates, without the parameter. The equation is as follows:

$$
\frac{d^{2} y}{d x^{2}}=\Gamma_{y y}^{x}\left(\frac{d y}{d x}\right)^{3}+\left(2 \Gamma_{x y}^{x}-\Gamma_{y y}^{y}\right)\left(\frac{d y}{d x}\right)^{2}+\left(\Gamma_{x x}^{x}-2 \Gamma_{x y}^{y}\right) \frac{d y}{d x}-\Gamma_{x x}^{y} .
$$

A general projective structure is therefore defined by a second-order ODE (1.5). In fact, two of the four functions $A_{0}, A_{1}, A_{2}, A_{3}$ can be eliminated by a coordinate transformation $(x, y) \rightarrow(\hat{x}(x, y), \hat{y}(x, y))$ which introduces two arbitrary functions.

On $T U$, the horizontal lifts of $\partial / \partial s^{i}$ are defined by

$$
S_{i}=\frac{\partial}{\partial s^{i}}-\Gamma_{i k}^{j} v^{k} \frac{\partial}{\partial v^{j}}
$$

where $v^{i}, i=1,2$ are the fibre coordinates of $T U$. The geodesics on $U$ lift to integral curves of the following spray on $T U$ :

$$
\Theta=v^{i} S_{i}=v^{i} \frac{\partial}{\partial s^{i}}-\Gamma_{j k}^{i} v^{j} v^{k} \frac{\partial}{\partial v^{i}}
$$

Now $\Theta$ is homogeneous of degree 1 in the $v^{i}$, so it projects to a section of a one dimensional distribution on PTU. PTU is the quotient of $T U-\{0$-section $\}$ by the vector field $v^{i} \frac{\partial}{\partial v^{i}}$. If $\lambda$ is a standard coordinate on one patch of the $\mathbb{R P}^{1}$ factor ${ }^{1}$, then the spray has the form

$$
\Theta=\partial_{x}+\lambda \partial_{y}+\left(A_{0}(x, y)+\lambda A_{1}(x, y)+\lambda^{2} A_{2}(x, y)+\lambda^{3} A_{3}(x, y)\right) \partial_{\lambda} .
$$

There is a unique curve in any direction through a point in $U$, which is why the curves can be lifted to a foliation of the projective tangent bundle $U \times \mathbb{R P}^{1}$.

\footnotetext{
${ }^{1}$ By standard coordinates $\lambda, \tilde{\lambda}$ on $\mathbb{R P}^{1}$ or $\mathbb{C P}^{1}$, we mean the usual coordinates $v^{1} / v^{0}$ and $v^{0} / v^{1}$, where $v^{0}, v^{1}$ are homogeneous coordinates.
} 
To obtain (3.1) we argue as follows. If $\tilde{\Theta}$ is the spray corresponding to a different connection $\tilde{\Gamma}$, then $\Gamma$ and $\tilde{\Gamma}$ are in the same projective class if $\Theta$ and $\tilde{\Theta}$ push down to the same spray on $P T U$. This gives

$$
\Theta-\tilde{\Theta} \propto v^{i} \frac{\partial}{\partial v^{i}}
$$

from which (3.1) follows, using the fact that the connections are torsion-free (i.e. symmetric in their lower indices).

\subsection{The twistor space of a projective structure}

Now suppose we have a holomorphic projective structure on a local patch of $\mathbb{C}^{2}$, which we still denote $U$. All of the above is still valid, with real coordinates replaced by complex ones. The functions $\Gamma_{j k}^{i}$ are now required to be holomorphic functions of the coordinates. Given a real-analytic projective structure, one can complexify by analytic continuation to obtain a holomorphic projective structure that will come equipped with a reality structure (see below).

The space $P T U$ is obtained from $T U$ on quotienting by $\mu^{i} \frac{\partial}{\partial \mu^{i}}$, which defines a tautological line bundle $\mathcal{O}(-1)$ over $P T U$.

As the $S_{i}$ are weight zero in the $\mu^{i}$ coordinates, they push down to vector fields on $P T U$, giving a two-dimensional distribution $\mathcal{S}$. Since $\Theta$ is weight one in the $\mu^{i}$, one must divide by a homogeneous polynomial of degree one in the $\mu^{i}$ to get something that pushes down to a vector field on PTU. The resulting vector field will have a singularity at a single point on each fibre, where the degree one polynomial vanishes. Different choices of polynomial will result in different vector fields on $P T U$, but they will always be in the same direction. In other words, $\Theta$ defines a one dimensional distribution which we shall call $D_{\Theta}$. Restricting to a $\mathbb{C P}^{1}$ fibre, $D_{\Theta}$ defines a line bundle over $\mathbb{C P}^{1}$. A section of this line bundle corresponds to a vector field in $D_{\Theta}$, i.e. a choice of polynomial as described above, and has a pole at a single point. Therefore by the classification of holomorphic line bundles over $\mathbb{C P}^{1}$, it must be $\mathcal{O}(-1)^{\dagger}$.

Restricting to a $\mathbb{C P}^{1}$ fibre, one obtains the following exact sequence of vector bundles over $\mathbb{C P}^{1}$

$$
0 \rightarrow \mathcal{O}(-1) \rightarrow \mathcal{O} \oplus \mathcal{O} \rightarrow \mathcal{S} / D_{\Theta} \rightarrow 0
$$

\footnotetext{
${ }^{\dagger}$ Coordinatize $\mathbb{C P}^{1}$ using two patches, $\mathcal{U}_{0}$ with coordinate $\lambda \in \mathbb{C}$, and $\mathcal{U}_{1}$ with coordinate $\eta \in \mathbb{C}$, and transition function $\lambda=1 / \eta$. The holomorphic line bundle $\mathcal{O}(n)$ over $\mathbb{C P}^{1}$ is defined by the transition function $a=\lambda^{n} b$ where $a \in \mathbb{C}$ is the fibre coordinate over $\mathcal{U}_{0}$ and $b(\eta) \in \mathbb{C}$ is the fibre coordinate over $\mathcal{U}_{1}$. The Birkhoff-Grothendieck theorem states that any holomorphic line bundle over $\mathbb{C P}^{1}$ is $\mathcal{O}(n)$ for some $n$. A global section of $\mathcal{O}(n)$ has $|n|$ zeroes or poles, for $n$ positive or negative respectively.
} 
where the first bundle is $D_{\Theta}$, the second is $\mathcal{S}$, and the last is the quotient. In fact, the quotient is $\mathcal{O}(1)$, for the following reason. Consider for instance the push down of $S_{0}$ to $P T U$. This defines a subbundle of $\mathcal{S}$ that is different to $\Theta$ everywhere except at a single point, the image of $\mu^{1}=0$. Hence it determines a section of $\mathcal{S} / \Theta$ which vanishes at a single point. Therefore, again using the classification of holomorphic line bundles over $\mathbb{C P}^{1}$, we have $\mathcal{S} / D_{\Theta} \cong \mathcal{O}(1)$.

The twistor space $Z$ is the two dimensional quotient of $P T U$ by $D_{\Theta}$. A point $u \in U$ corresponds to a twistor line $\hat{u} \subset Z$ corresponding to all the geodesics through $u$. The normal bundle of an embedded $\hat{u}=\mathbb{C P}^{1} \subset Z$ is given by the quotient bundle in the above sequence, i.e. $\mathcal{O}(1)$. This is summarized by a double fibration picture:

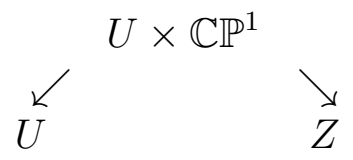

The left arrow denotes projection to $U$, and the right arrow denotes the quotient by $D_{\Theta}$.

The converse is also valid:

Theorem 3. [11, 17] There is a 1-1 correspondence between local two dimensional holomorphic projective structures and complex surfaces containing an embedded $\mathbb{C P}^{1}$ with normal bundle $\mathcal{O}(1)$.

A vector $V \in T_{u} U$ corresponds to a global section of the normal bundle $\mathcal{O}(1)$ of $\hat{u}$. Such a section vanishes at a single point $p \in Z$. The geodesic of the projective structure through this direction is given by points in $U$ corresponding to twistor lines in $Z$ that intersect $\hat{u}$ at $p$. That there is a one-parameter family of such lines can be shown by blowing up $Z$ at the vanishing point and using Kodaira theory, see [1].

\subsection{Flatness of projective structures}

A projective structure is said to be flat if the corresponding second order ODE (1.5) can be transformed to the trivial ODE

$$
\frac{d^{2} y}{d x^{2}}=0
$$

by coordinate transformation $(x, y) \rightarrow(\hat{x}(x, y), \hat{y}(x, y))$. The terminology comes from the fact that given any second order ODE one can construct a Cartan connection on a certain $G$-structure [2], and when this connection is flat the equation can be transformed to the trivial ODE (3.6). It turns out that a second order ODE must be 
of the form (1.5) to be flat, and in addition the functions $A_{0}, A_{1}, A_{2}, A_{3}$ must satisfy some PDEs. Defining

$$
F(x, y, \lambda)=A_{0}(x, y)+\lambda A_{1}(x, y)+\lambda^{2} A_{2}(x, y)+\lambda^{3} A_{3}(x, y)
$$

the following must hold [2]:

$$
\frac{\mathrm{d}^{2}}{\mathrm{~d} x^{2}} F_{11}-4 \frac{\mathrm{d}}{\mathrm{d} x} F_{01}-F_{1} \frac{\mathrm{d}}{\mathrm{d} x} F_{11}+4 F_{1} F_{01}-3 F_{0} F_{11}+6 F_{00}=0
$$

where

$$
F_{0}=\frac{\partial F}{\partial y}, \quad F_{1}=\frac{\partial F}{\partial \lambda}, \quad \frac{\mathrm{d}}{\mathrm{d} x}=\frac{\partial}{\partial x}+\lambda \frac{\partial}{\partial y}+F \frac{\partial}{\partial \lambda}
$$

This is a set of PDEs for the functions $A_{0}, A_{1}, A_{2}, A_{3}$.

\subsection{Reality conditions for projective structures}

A reality structure for $Z$ is an anti-holomorphic involution that leaves invariant a two real parameter family of twistor lines, and fixes an equator of each line. Given a line in this real family, all the sections pointing to nearby lines in the real family have a zero at some point, and the union of these points gives an equator of the line; this equator must be fixed by the reality structure. The real family of twistor lines then corresponds to a real manifold $U$ with a projective structure.

In this paper all holomorphic projective structure have reality structures since they occur as complexifications of real projective structures.

\section{Null Killing Vectors and Twistor Space}

\subsection{The twistor space of an ASD conformal structure}

In the following and for the rest of the paper, $\tilde{\mathbf{e}}_{A A^{\prime}}$ denote the horizontal lifts of $\mathbf{e}_{A A^{\prime}}$ to $S^{\prime}$, or their push-down to $P S^{\prime}$. Abstractly, the integral curves of these horizontal lifts define parallely transported primed spinors using the connection on $S^{\prime}$ (see Appendix (A).

We can abstractly define the two-dimensional twistor distribution on $S^{\prime}$ as follows. A point $s \in S^{\prime}$ is determined by a primed spinor $\pi$ at a point $x \in M$. The null vectors $\pi \otimes \mu$ for all unprimed spinors $\mu$ span an $\alpha$-plane at $x$. Define the twistor distribution at $s$ to be the subspace of horizontal vectors at $s$ whose push-down to the base lies in this $\alpha$-plane. 
Concretely, the twistor distribution is spanned by vectors $L_{A}(A=0,1)$ on $S^{\prime}$, defined with a choice of tetrad by

$$
L_{A}=\pi^{A^{\prime}} \tilde{\mathbf{e}}_{A A^{\prime}}=\pi^{A^{\prime}}\left(\mathbf{e}_{A A^{\prime}}-\Gamma_{A A^{\prime} B^{\prime}}^{C^{\prime}} \pi^{B^{\prime}} \frac{\partial}{\partial \pi^{C^{\prime}}}\right),
$$

where $\pi^{A^{\prime}}$ are the local coordinates on the fibres of $S^{\prime}$. In the Appendix it is shown that the twistor distribution is integrable for ASD conformal structures, which is a seminal result of Penrose 21]. In other words, given a neutral ASD conformal structure $[g]$, each self-dual two plane at a point is tangent to a unique $\alpha$-surface through that point, which is the push down of a leaf of the twistor distribution. In the holomorphic case, the space of leaves of the twistor distribution (locally, over a suitably convex region of the base), is a three dimensional complex manifold $\mathcal{P} \mathcal{T}$ called the twistor space [21, 11].

The double fibration picture is very similar to the projective structure case discussed in Section 3.1. The projective primed spin bundle $P S^{\prime}$ is the quotient of $S^{\prime}$ by the vector field $\pi^{A^{\prime}} \frac{\partial}{\partial \pi^{A^{\prime}}} . P S^{\prime}$ is fundamental in the fibration picture, as each $\alpha$ surfacein $M$ has a unique lift, in the same way that each geodesic of a projective structure has a unique lift to the projective tangent bundle. The horizontal vectors $\tilde{\mathbf{e}}_{A A^{\prime}}$ are weight zero in the $\pi^{A^{\prime}}$ coordinates, so push down to vector fields on $P S^{\prime}$, giving a four-dimensional distribution $\Xi$ on $P S^{\prime}$. The $L_{A}$ vectors (4.1) are weight one, so together define a two dimensional subdistribution $\mathcal{L}$ of $\Xi$, which restricts to $\mathcal{O}(-1) \oplus \mathcal{O}(-1)$ on a $\mathbb{C P}^{1}$ fibre; we also refer to this as the twistor distribution. Over a $\mathbb{C P}^{1}$ fibre, there is an exact sequence

$$
0 \rightarrow \mathcal{O}(-1) \oplus \mathcal{O}(-1) \rightarrow \mathcal{O} \otimes \mathbb{C}^{4} \rightarrow \Xi / \mathcal{L} \rightarrow 0
$$

The first term is $\mathcal{L}$, the second is $\Xi$. As in the projective structure case, one can show that $\Xi / \mathcal{L}$ is $\mathcal{O}(1) \oplus \mathcal{O}(1)$. The twistor space $\mathcal{P} \mathcal{T}$ is the quotient of $P S^{\prime}$ by $\mathcal{L}$. The image of a $\mathbb{C P}^{1}$ fibre over $x \in M$ is an embedded $\mathbb{C P}^{1} \in \mathcal{P} \mathcal{T}$, and has normal bundle $\mathcal{O}(1) \oplus \mathcal{O}(1)$, the quotient bundle in (4.2). It corresponds to all the $\alpha$-surfaces through $x$.

The twistor correspondence is summarized by the double fibration:

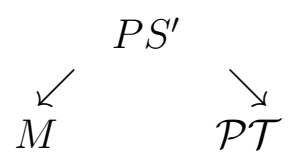

Here the left arrow denotes projection to $M$, and the right arrow denotes the quotient by $\mathcal{L}$.

Again, there is a converse: 
Theorem. (Penrose [21]) There is a 1-1 correspondence between local four dimensional holomorphic ASD conformal structures $(M,[g])$ and three dimensional complex manifolds $\mathcal{P} \mathcal{T}$ with an embedded $\mathbb{C P}^{1} \subset \mathcal{P} \mathcal{T}$, with normal bundle $\mathcal{O}(1) \oplus \mathcal{O}(1)$.

The essential fact is that an embedded $\mathbb{C P}^{1}$ with the above normal bundle belongs to a family of embedded $\mathbb{C P}^{1}$ s parameterized by a complex 4 -manifold $M$. Vectors at $x \in M$ correspond to sections of the normal bundle of $\hat{x}$, and null vectors are given by sections with a zero. This defines a conformal structure, because a global section of $\mathcal{O}(1) \oplus \mathcal{O}(1)$ is given by $(a \lambda+b, c \lambda+d)$ for affine coordinate $\lambda \in \mathbb{C},(a, b, c, d) \in \mathbb{C}^{4}$, and this can only be $(0,0)$ when $a d-b c=0$, which is a quadratic condition. In this case there is a zero at a single point. The conformal structure is anti-self-dual, with $\alpha$-surfaces defined by families of twistor lines through a fixed point in $\mathcal{P} \mathcal{T}$.

In this picture, the $\alpha$-surfaces are obtained as follows. Let $\hat{x} \subset \mathcal{P} \mathcal{T}$ be the twistor line corresponding to a point $x \in M$. Let $V \in T_{x} M$ be a null vector. We want to show that $V$ lies in a unique $\alpha$-surface through $x$. The corresponding section of the normal bundle of $\hat{x}$ has a zero at some point $p \in \mathcal{P} \mathcal{T}$ because $V$ is null. The $\alpha$-surface corresponds to all the twistor lines that intersect $\hat{x}$ at $p$. There is a two-parameter family of such lines. It is easy to see that there is a two-parameter family of sections that vanish at $p$. To show that these are tangent to a two-parameter family of lines one must blow-up $\mathcal{P} \mathcal{T}$ at $p$ and use Kodaira theory; see [11] for details.

\subsubsection{Reality conditions for split signature}

In order to obtain a real split signature metric from a twistor space, we must be able to distinguish a four real parameter family of twistor lines, whose parameter space will be the four real dimensional manifold. In addition we require that given a line in this real family, the sections of the normal bundle that point to others in the family inherit a split signature conformal structure. As described above, a section of $\mathcal{O}(1) \oplus \mathcal{O}(1)$ is defined by four complex numbers $(a, b, c, d)$, with a quadratic form defined by $a d-b c$. If we restrict $(a, b, c, d)$ to be real we obtain a real split signature conformal structure. The sections tangent to the real family are of this type.

The zero of such sections occurs for real $\lambda$, that is, on an equator of $\mathbb{C P}^{1}$. The conformal structure is thus invariant under an anti-holomorphic involution of the $\mathbb{C P}^{1}$ that has the equator fixed. A split signature real structure on $\mathcal{P} \mathcal{T}$ is an antiholomorphic involution that leaves invariant a four real parameter family of twistor lines, and when restricted to one of these fixes an equator.

Not all holomorphic metrics have real structures, but all the holomorphic metrics in this paper have obvious real 'slices' because they are complexifications of real metrics, obtained by letting the real coordinates be complex. 


\subsection{Lift of $K$ to $P S^{\prime}$}

Now given a null conformal Killing vector for an ASD conformal structure, the fact that $M$ is foliated by $\alpha$-surfaces (Lemma 1) is not very illuminating, since they must already exist by anti-self-duality. The foliation by $\beta$-surfaces is more interesting, since these do not exist generically.

In this section we will prove that in the analytic case, the space of $\beta$-surfaces inherits a natural projective structure. We then explain how this arises geometrically, due to the presence of $\alpha$-surfaces ensured by anti-self-duality.

Let $K$ be a null conformal Killing vector for $(M,[g])$. We assume $K$ is without fixed points, which can always be arranged by restricting $M$ to a suitable open set.

Since $K$ preserves the conformal structure, the corresponding diffeomorphism maps $\alpha$-surfaces to $\alpha$-surfaces, and hence it induces a vector field $\mathcal{K}$ on $\mathcal{P} \mathcal{T}$. We now translate this fact into a statement on the projective primed spin bundle $P S^{\prime}$. Each $\alpha$-surface has a unique lift and these lifts foliate $P S^{\prime}$. The following proposition shows how to lift $K$ to $P S^{\prime}$, giving a vector field that is Lie-derived along the lifts of the $\alpha$-surfaces.

Proposition 1. Let $K=K^{A A^{\prime}} \boldsymbol{e}_{A A^{\prime}}$ be a conformal Killing vector for an ASD metric g. Define a vector field $\tilde{K}$ on $S^{\prime}$ by

$$
\tilde{K}:=K^{A A^{\prime}} \tilde{\boldsymbol{e}}_{A A^{\prime}}+\pi_{A^{\prime}} \phi^{A^{\prime} B^{\prime}} \frac{\partial}{\partial \pi^{B^{\prime}}}+\frac{1}{2} \eta \pi^{A^{\prime}} \frac{\partial}{\partial \pi^{A^{\prime}}} .
$$

Then this satisfies

$$
\left[\tilde{K}, L_{A}\right]=\left(K^{B B^{\prime}} \Gamma_{B B^{\prime} A}^{D}-\psi_{A}{ }^{D}\right) L_{D}+\frac{3}{4}\left(\boldsymbol{e}_{A B^{\prime}} \eta\right) \pi^{B^{\prime}} \pi^{C^{\prime}} \frac{\partial}{\partial \pi^{C^{\prime}}}
$$

Proof. See Appendix.

Remark. Since $\tilde{K}$ is weight zero in the $\pi^{A^{\prime}}$ coordinates, it defines a vector field on $P S^{\prime}$, which we will also refer to as $\tilde{K}$ by abuse of notation. The last term on the right hand side of (4.4) is proportional to the Euler vector field, so does not contribute to $\tilde{K}$ on $P S^{\prime}$. Hence (4.4) shows that $\tilde{K}$ commutes with the twistor distribution $\mathcal{L}$ on $P S^{\prime}$. The vector field $\mathcal{K}$ on $\mathcal{P} \mathcal{T}$ is the push-forward of $\tilde{K}$ to $\mathcal{P} \mathcal{T}$, which is well defined because $\tilde{K}$ is Lie-derived along $\mathcal{L}$.

\subsection{Projective structure from a quotient}

In this section we assume that $[g]$ is analytic, so we can complexify by analytic continuation. Thus we are now working on a local patch of $\mathbb{C}^{4}$, with a holomorphic 
conformal structure. We assume that we have restricted to a suitable open set on the base so that all the spaces of leaves involved are non-singular complex manifolds.

As in Section 2, write $K=\iota^{A} O^{A^{\prime}} \mathbf{e}_{A A^{\prime}}$, where now $\mathbf{e}_{A A^{\prime}}$ is a holomorphic tetrad and $\iota^{A}, O^{A^{\prime}}$ are complex spinor fields that vary holomorphically.

When $K$ is null, it is easy to see that $\mathcal{K}$, the induced vector field on twistor space $\mathcal{P} \mathcal{T}$, will vanish on a hypersurface $\mathcal{H}$ in $\mathcal{P} \mathcal{T}$, because $K$ fixes a two-parameter family of $\alpha$-surfaces, which are those to which it is tangent. These are the 'special' $\alpha$-surfaces of Lemma 1. We now explain how this is seen from the lift $\tilde{K}$ to $P S^{\prime}$.

On $S^{\prime}, \tilde{K}$ from Proposition 1 is given by:

$$
\tilde{K}=\iota^{A} O^{A^{\prime}} \tilde{\mathbf{e}}_{A A^{\prime}}+\pi_{A^{\prime}} \phi^{A^{\prime} B^{\prime}} \frac{\partial}{\partial \pi^{B^{\prime}}}+\frac{1}{2} \eta \pi^{A^{\prime}} \frac{\partial}{\partial \pi^{A^{\prime}}} .
$$

Now when $\pi^{A^{\prime}} \propto o^{A^{\prime}}$, one has $\pi_{A^{\prime}} \phi^{A^{\prime} B^{\prime}} \propto o^{B^{\prime}}$ from (2.5), so the second term on the RHS is proportional to the Euler vector field $\Upsilon=\pi^{A^{\prime}} \frac{\partial}{\partial \pi^{A^{\prime}}}$. The last term is everywhere proportional to the Euler vector field. To go from $S^{\prime}$ to $P S^{\prime}$ one quotients $S^{\prime}-\{0$-section $\}$ by the integral curves of $\Upsilon$. So we have shown that on the section $\left[\pi^{A^{\prime}}\right]=\left[o^{A^{\prime}}\right]$ of $P S^{\prime}, \tilde{K}$ is the push down of $\iota^{A} O^{A^{\prime}} \tilde{\mathbf{e}}_{A A^{\prime}}$ only. But this is in $\mathcal{L}$, so $\tilde{K}$ pushes down to the zero vector under the quotient of $P S^{\prime}$ by $\mathcal{L}$.

So there is a (complex) hypersurface in $P S^{\prime}$, defined by the section $\left[\pi^{A^{\prime}}\right]=\left[o^{A^{\prime}}\right]$, on which $\tilde{K}$ lies in the twistor distribution. One can also define this hypersurface as the image in $P S^{\prime}$ of the hypersurface $\pi . o=0$ in $S^{\prime}$, under the quotient by $\Upsilon$. We will refer to this hypersurface as $H$. It is easy to see by pushing down to the base that $\tilde{K}$ is linearly independent of the twistor distribution everywhere else on $P S^{\prime}$.

Define a vector field

$$
V=\iota^{A} L_{A}=\iota^{A} \pi^{A^{\prime}} \tilde{\mathbf{e}}_{A A^{\prime}}
$$

on $S^{\prime}$. This is weight one in the $\pi^{A^{\prime}}$ coordinates, so gives a one dimensional distribution on $P S^{\prime}$ which restricts to $\mathcal{O}(-1)$ on fibres. Together with $\operatorname{span}\{\tilde{K}\}$, we get a two dimensional distribution on $P S^{\prime}-H$. On $H$, the distribution drops its rank from two to one.

The two dimensional distribution defined by $\{V, \tilde{K}\}$ on $P S^{\prime}-H$ pushes down to the $\beta$-plane distribution $\mathcal{D}_{\beta}$ on the base.

Lemma 2. The two dimensional distribution on $P S^{\prime}-H$ determined by $\{V, \tilde{K}\}$ is integrable.

Proof. We work on $S^{\prime}$ for convenience, and push down to $P S^{\prime}$ at the end. The distribution $\operatorname{span}\{\tilde{K}, V\}$ on $S^{\prime}$ is two dimensional on $S^{\prime}$ when $\pi^{A^{\prime}} o_{A^{\prime}} \neq 0$. Multiples 
of the Euler field $\Upsilon$ are therefore irrelevant.

$$
\begin{aligned}
{[V, \tilde{K}]=} & {\left[\tilde{K}, \iota^{C} L_{C}\right] } \\
= & \iota^{C}\left[\tilde{K}, L_{C}\right]+\tilde{K}\left(\iota^{B}\right) L_{B} \\
= & \iota^{C}\left(\left(K^{B B^{\prime}} \Gamma_{B B^{\prime} C}{ }^{D}-\psi_{C}{ }^{D}\right) L_{D}+\frac{3}{4}\left(\mathbf{e}_{C B^{\prime}} \eta\right) \pi^{B^{\prime}} \pi^{C^{\prime}} \frac{\partial}{\partial \pi^{C^{\prime}}}\right) \\
& \quad+K^{B B^{\prime}} \mathbf{e}_{B B^{\prime}}\left(\iota^{C}\right) L_{C} \\
= & \left(K^{B B^{\prime}} \nabla_{B B^{\prime}} \iota^{C}-\iota^{D} \psi_{D}{ }^{C}\right) L_{C}+\# \Upsilon \\
= & \left(\iota^{B} o^{B^{\prime}} \nabla_{B B^{\prime}} \iota^{C}-\iota^{D} \psi_{D}{ }^{C}\right) L_{C}+\# \Upsilon .
\end{aligned}
$$

From (2.5) we have $\iota^{D} \psi_{D}^{C} \propto \iota^{C}$, and from (2.7) we have $\iota^{B} O^{B^{\prime}} \nabla_{B B^{\prime}} \iota^{C} \propto \iota^{C}$. Hence the RHS is proportional to $V$, ignoring the irrelevant Euler vector field part.

Next we will show that it is possible to continue this distribution over the hypersurface $H$ so it is rank two on the whole of $P S^{\prime}$, and that the resulting distribution commutes on the hypersurface. It will then be possible to quotient $P S^{\prime}$ by the leaves of this distribution.

Lemma 3. There is a two-dimensional integrable distribution $\mathcal{D}$ over $P S^{\prime}$, which on $P S^{\prime}-H$ is determined by $\{\tilde{K}, V\}$. Let $\varrho$ be the projection $P S^{\prime} \rightarrow M$. Then for every $p \in P S^{\prime}$, we have $\varrho_{*}\left(\left.\mathcal{D}\right|_{p}\right)=\mathcal{D}_{\beta}$.

Remark. Intuitively one can think of $\mathcal{D}$ as a lift of the $\beta$-surfaces to $P S^{\prime}$, where each $\beta$-surface has a $\mathbb{C P}^{1}$ of lifts.

Proof. Choose a spinor $\iota^{A^{\prime}}$ satisfying $o^{A^{\prime}} \iota_{A^{\prime}}=1$. Define the following (singular) vector field on $S^{\prime}$ :

$$
W=\frac{1}{\pi^{C^{\prime}} o_{C^{\prime}}}\left(V-\left(\pi^{D^{\prime}} \iota_{D^{\prime}}\right) \tilde{K}\right)
$$

This is weight zero in the $\pi^{A^{\prime}}$, so defines a vector field on $P S^{\prime}$ by push-forward, which we shall also call $W$. We will now show that $W$ is well defined even over $H \subset P S^{\prime}$, despite the $1 /\left(\pi^{C^{\prime}} o_{C^{\prime}}\right)$ factor in (4.5).

Without loss of generality, choose a tetrad such that

$$
K=\iota^{A} O^{A^{\prime}} \mathbf{e}_{A A^{\prime}}=\mathbf{e}_{00^{\prime}} .
$$

That is, $\iota^{A}=(1,0), o^{A^{\prime}}=(1,0)$. Define $\lambda=\pi^{1^{\prime}} / \pi^{0^{\prime}}$ to be the coordinate on the $\pi^{0^{\prime}} \neq 0$ patch of $\mathbb{C P}^{1}$, and extend this to a patch of $P S^{\prime}$; we call the patch $\mathcal{U}$. Then $H$ lies entirely within $\mathcal{U}$ at $\lambda=0$. We have the following expression for $\tilde{K}$, obtained by 'projectivizing' (4.3):

$$
\begin{aligned}
\tilde{K} & =\tilde{\mathbf{e}}_{00^{\prime}}+\left(\phi_{0^{\prime}}{ }^{1^{\prime}}+\lambda\left(\phi_{1^{\prime}}{ }^{1^{\prime}}-\phi_{0^{\prime}}{ }^{0^{\prime}}\right)+\lambda^{2} \phi_{1^{\prime}}{ }^{0^{\prime}}\right) \frac{\partial}{\partial \lambda} \\
& =\tilde{\mathbf{e}}_{00^{\prime}}+\left(\lambda\left(\phi_{1^{\prime}}{ }^{\prime}-\phi_{0^{\prime}}{ }^{0^{\prime}}\right)+\lambda^{2} \phi_{1^{\prime}}{ }^{{ }^{\prime}}\right) \frac{\partial}{\partial \lambda}
\end{aligned}
$$


where $\phi_{0^{\prime}}{ }^{1^{\prime}}=0$ due to (2.6) $)$.

In the above conventions, we have $V=\pi^{A^{\prime}} \tilde{\mathbf{e}}_{0 A^{\prime}}$. On $\mathcal{U} \subset P S^{\prime}$, the push forward of $\frac{1}{\pi^{C^{\prime}} o_{C^{\prime}}} V$ is

$$
\frac{1}{\lambda} \tilde{\mathbf{e}}_{00^{\prime}}+\tilde{\mathbf{e}}_{01^{\prime}},
$$

which is singular at $H$, corresponding to $\lambda=0$. Choosing $\iota^{A^{\prime}}=(0,-1)$, we then obtain the following expression for $W$ on $\mathcal{U}$ :

$$
\left.W=\frac{1}{\lambda} \tilde{\mathbf{e}}_{00^{\prime}}+\tilde{\mathbf{e}}_{01^{\prime}}-\frac{1}{\lambda} \tilde{K}=\tilde{\mathbf{e}}_{01^{\prime}}-\left(\left(\phi_{1^{\prime}}{ }^{1^{\prime}}-{\phi_{0}}_{0^{\prime}}{ }^{0^{\prime}}\right)+\lambda \phi_{1^{\prime}}{ }^{0^{\prime}}\right)\right) \frac{\partial}{\partial \lambda} .
$$

This is a non-singular vector field on $\mathcal{U}$. By construction, away from $H$ this lies in $\operatorname{span}\{\tilde{K}, \tilde{V}\}$. Define $\mathcal{D}$ on $\mathcal{U}$ to be $\operatorname{span}\{\tilde{K}, W\}$. This is clearly non-degenerate everywhere on $\mathcal{U}$. Note that $W$ is also well defined over the other patch (i.e. at $\lambda=\infty)$ so we can define $\mathcal{D}$ as $\operatorname{span}\{\tilde{K}, W\}$ over the whole of $P S^{\prime}$.

We now want to show that $\mathcal{D}$ is integrable over $H$. We know (Lemma 21) that $\mathcal{D}$ is integrable away from $H$. Therefore on $\mathcal{U}$ we have

$$
[\tilde{K}, W]=f \tilde{K}+g W+Y,
$$

where $f, g$ are holomorphic functions on $\mathcal{U}$ and $Y$ is a holomorphic vector field vanishing on $\mathcal{U}-H$. But such a vector field must vanish, otherwise it is not even continuous, so is certainly not holomorphic.

The last part of the lemma is obvious, just from inspecting the coordinate expressions of $\tilde{K}, W$.

We now have a three dimensional integrable distribution $\mathcal{L} \cup \mathcal{D}$. It is three dimensional because at each point $\mathcal{L}$ and $\mathcal{D}$ have a direction in common, which is the one-dimensional distribution defined on $P S^{\prime}$ by the push-forward of $V$ on $S^{\prime}$. From Lemma 3, $\mathcal{D}$ is an integrable subdistribution. Note that $\mathcal{D}$ consists of a $\mathbb{C P}^{1}$ of lifts of each $\beta$-surface in the base. If we pick a suitably convex set on the base so that the space of $\beta$-surfaces $U$ intersecting it is a Hausdorff complex manifold, then the quotient $P S^{\prime} / \mathcal{D}$ will also be a Hausdorff complex manifold. A point in this quotient is a point in $U$ together with a choice of lift.

In fact we can canonically identify $P S^{\prime} / \mathcal{D}$ with $P T U$, the projective tangent bundle of $U$, as follows. Using the conventions of Lemma 3. the tangent planes to the $\beta$-surfaces in the base are spanned at each point by $\mathbf{e}_{00^{\prime}}, \mathbf{e}_{01^{\prime}}$. Now $L_{1}$ has the form $\mathbf{e}_{10^{\prime}}+\lambda \mathbf{e}_{11^{\prime}}+(\ldots) \partial_{\lambda}$, so at each point in the fibre above a point $x \in M, L_{1}$ pushes down to a different null direction transverse to the $\beta$-plane at $x$. Now suppose we take a lift of a $\beta$-surface $\Pi$, i.e. a leaf of $\mathcal{D}$ that projects down to $\Pi$. Push down $L_{1}$ at each point over this lift. This will give a vector field $\Theta=\mathbf{e}_{10^{\prime}}+\lambda \mathbf{e}_{11^{\prime}}$ over $\Pi$, where $\lambda$ is now a function on the $M$. 
We want to show that this determines a projective vector at the point $s \in U$ corresponding to $S$. This means we require $\left[\mathbf{e}_{00^{\prime}}, \Theta\right] \propto \Theta \bmod \left\{\mathbf{e}_{00^{\prime}}, \mathbf{e}_{01^{\prime}}\right\},\left[\mathbf{e}_{01^{\prime}}, \Theta\right] \propto$ $\Theta \bmod \left\{\mathbf{e}_{00^{\prime}}, \mathbf{e}_{01^{\prime}}\right\}$. But it is easy to show that this is satisfied, using the fact that the distribution spanned by $\tilde{K}, W, L_{1}$ commutes. Hence to determine the projective vector corresponding to a leaf of $\mathcal{D}$, just choose a point on the leaf and push down $L_{1}$. Because of the above considerations, this direction will be independent of the choice of point on the leaf.

Proof of Theorem $\mathbb{1}$. Define $Z$ as the quotient of $P S^{\prime}$ by $\mathcal{L} \cup \mathcal{D}$. Equivalently, this is the quotient of $\mathcal{P} \mathcal{T}$ by a one-dimensional distribution which on $\mathcal{P} \mathcal{T}-\mathcal{H}$ is $\operatorname{span}\{\mathcal{K}\}$. The image of a $\mathbb{C P}^{1}$ fibre of $P S^{\prime}$ under the quotient is a twistor line in $Z$.

On a $\mathbb{C P}^{1}$ fibre, the horizontal part of $\mathcal{D}$ defines a subbundle $\mathcal{O} \otimes \mathbb{C}^{2}$ of the horizontal distribution $\Xi=\mathcal{O} \otimes \mathbb{C}^{4}$, corresponding to the horizontal parts of the vectors $\tilde{K}$ and $W$. Choosing a spinor $o^{A}$ such that $\iota^{A} O_{A}=1$, we can form the vector field $o^{A} L_{A}$ on $S^{\prime}$, which pushes down to a horizontal distribution on $P S^{\prime}$ that is always linearly independent of $\mathcal{D}$. Since the $L_{A}$ are weight one, this is $\mathcal{O}(-1)$ when restricted to a $\mathbb{C P}^{1}$ fibre. Because $\mathcal{L} \cup \mathcal{D}$ is integrable (Lemma 3 ), this distribution determines a one dimensional distribution $D_{\Theta}$ on $P T U=P S^{\prime} / \mathcal{D}$. The spray $\Theta$ of a projective structure is a section of $\mathcal{D}_{\Theta} \otimes \mathcal{O}(1)$ where here $\mathcal{O}(1)$ is dual to the tautological line bundle over PTU. The situation is described by the following commuting diagram:

$$
\begin{aligned}
& 0 \rightarrow \mathcal{O}(-1) \oplus \mathcal{O}(-1) \rightarrow \mathcal{O} \otimes \mathbb{C}^{4} \rightarrow \mathcal{O}(1) \oplus \mathcal{O}(1) \rightarrow 0 \\
& \downarrow V \\
& 0 \rightarrow \quad \mathcal{O}(-1) \\
& \downarrow \mathcal{D} \\
& \rightarrow \mathcal{O} \otimes \mathbb{C}^{2} \rightarrow \quad \mathcal{O}(1) \quad \rightarrow \quad 0
\end{aligned}
$$

where these are bundles over a $\mathbb{C P}^{1}$ fibre of $P S^{\prime}$. The vector field $o^{A} L_{A}$ on $S^{\prime}$ constructed above corresponds to the $\mathcal{O}(-1)$ in the bottom row after quotienting by $V$, and gives the projective structure spray. The bottom row is the sequence (3.5) on $P T U=P S^{\prime} / \mathcal{D}$, where $U$ is the space of $\beta$-surfaces in $M$. Thus there is a projective structure on $U$.

Remark. The real space of $\beta$-surfaces has a system of curves that comes from the quotienting operations described above but with real spaces instead of complex. These real curves are described by the holomorphic projective structure with a reality structure.

Figure 1 illustrates the situation. Here $p$ and $q$ are the obvious projections. $\mathcal{D}_{\beta}$ represents the $\beta$-surface distribution on $M$. The $\widehat{\mathcal{K}}$ labelling the map from $\mathcal{P} \mathcal{T}$ to $Z$ requires some of explanation. The vector field $\tilde{K}$ over $P S^{\prime}$ commutes with the twistor distribution (Lemma I), so determines a vector field $\mathcal{K}$ on $\mathcal{P} \mathcal{T}$. This vector field vanishes on a hypersurface $\mathcal{H} \subset \mathcal{P} \mathcal{T}$, corresponding to the $\alpha$-surfaces to which 


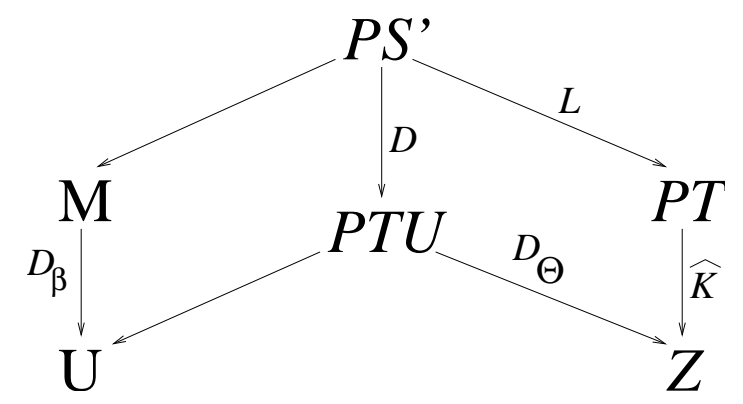

Figure 1: Relationship between foliation spaces.

$K$ is tangent; these are the $\alpha$-surfaces appearing in Lemma 1. Now $\mathcal{K}$ on $\mathcal{P} \mathcal{T}$ only depends on $\tilde{K}$ modulo $\mathcal{L}$. Lemma 3 shows that we can multiply $\tilde{K}$ modulo $\mathcal{L}$ by a meromorphic function $(1 / \lambda)$ and obtain a vector field $W$ commuting with the twistor distribution. This means that there is a one-dimensional distribution $\widehat{\mathcal{K}}$ over the whole of $\mathcal{P} \mathcal{T}$ that never degenerates, and which agrees with span $\{\mathcal{K}\}$ on $\mathcal{P} \mathcal{T}-\mathcal{H}$. The quotient of $\mathcal{P} \mathcal{T}$ by this distribution gives $Z$, as illustrated in the diagram.

One can rephrase this in terms of divisor line bundles. That is, there is a holomorphic line bundle $\mathcal{E}$ over $\mathcal{P} \mathcal{T}$ defined by the property that it has a meromorphic section $\zeta$ with a pole of order one on $\mathcal{H}$. Then $\zeta \otimes \mathcal{K}$ defines a non-vanishing section of $\mathcal{E} \otimes T \mathcal{P} \mathcal{T}$. This is equivalent to the one dimensional distribution $\widehat{\mathcal{K}}$ over $\mathcal{P} \mathcal{T}$ described above. To obtain the distribution one simply finds trivializations of $\mathcal{E}$ and $T \mathcal{P} \mathcal{T}$ over a patch, and expresses $\zeta$ in this trivialization. Its direction will be independent of the trivialization of $\mathcal{E}$, and defines the distribution over the patch.

\subsection{Relationship of the twistor spaces}

Here we discuss the relationship between the twistor spaces without the foliation space picture. Incidence relation between various objects in $M$ and $\mathcal{P} \mathcal{T}$ is represented by (Fig. 2).

First one must understand what a $\beta$-surface corresponds to in $\mathcal{P} \mathcal{T}$. The answer is a two-parameter family of twistor lines, each of which intersects any other at a single point. This is because all points on a $\beta$-surface are null separated. However, unlike the case of an $\alpha$-surface, there is not just a single point of intersection of the whole family. To construct the family, pick a point on the $\beta$-surface, say $x$. Then $\hat{x}$ is a twistor line in $\mathcal{P} \mathcal{T}$. Now $\mathcal{K}$ determines a section of the normal bundle with a zero. Twistor lines intersecting $\hat{x}$ at this zero are on the $\beta$-surface, and correspond to those along the trajectory of $K$ through $x$. In fact this is a null geodesic, since null Killing vector fields have geodesic integral curves. Now pick another section of the 
Figure 2: The $\alpha$ and $\beta$ surfaces in $M$ intesect along a trajectory of $K$ which is a null geodesic. This corresponds to a point $\alpha$ lying on a surface $\beta$ in $\mathcal{P} \mathcal{T}$. Points $p_{1}, p_{2}, p_{3}$ in $M$ correspond to projective lines in $\mathcal{P} \mathcal{T}$.
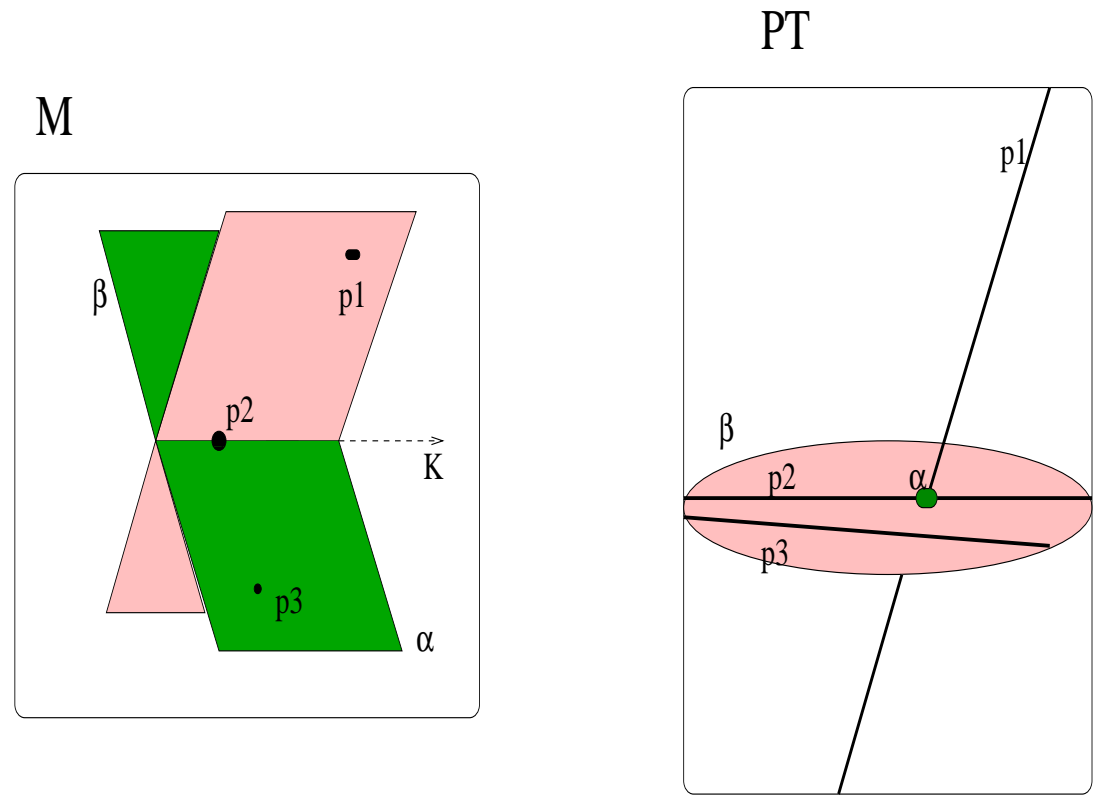

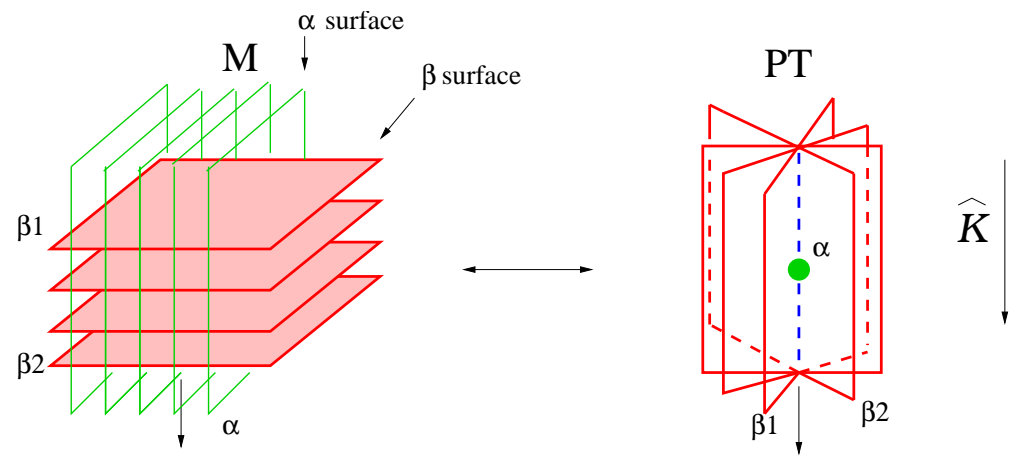

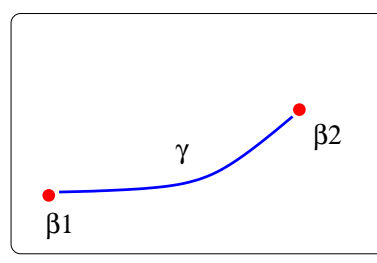

$\mathrm{U}$

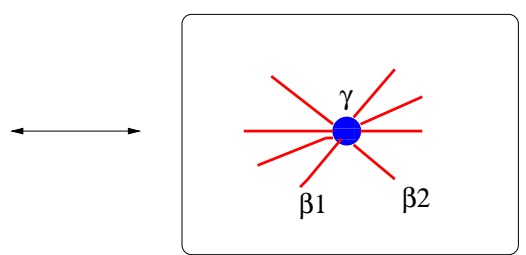

$\mathrm{Z}$

Figure 3: Relationship between $M, U, \mathcal{P} \mathcal{T}$ and $Z$.

normal bundle with a zero at a different point, such that all linear combinations of this with the section determined by $\mathcal{K}$ also have a zero. The resulting two dimensional distribution in $M$ at $x$ is a $\beta$-plane. Doing this for each $x \in M$ gives a $\beta$-plane distribution which is integrable.

The diagram (Fig. 4.4) illustrates the situation. In $M$, a one parameter family of $\beta$-surface is shown, each of which intersects a one parameter family of $\alpha$-surfaces, also shown. The $\beta$-surfaces correspond to a projective structure geodesic in $U$, shown at the bottom left.

The $\beta$-surfaces in $M$ correspond to surfaces in $\mathcal{P} \mathcal{T}$, as discussed above. These surfaces intersect at the dotted line, which corresponds to the one parameter family of $\alpha$-surfaces in $M$. When we quotient $\mathcal{P} \mathcal{T}$ by $\mathcal{K}$ to get $Z$, the surfaces become twistor lines in $Z$, and the dotted line becomes a point at which the twistor lines intersect; this is shown on the bottom right. This family of twistor lines intersecting at a point corresponds to the geodesic of the projective structure.

\section{Local classification}

The second theorem stated in the Introduction gives a local expression for any analytic neutral signature ASD conformal structure. We now prove this theorem. In the proof we will often use the following shorthand for coordinate transformations: $t \rightarrow$ 
$F(t, x, y, z)$ means define a new coordinate $\tilde{t}=F(t, x, y, z)$ and then relabel it $t$ again. This avoids having to introduce new symbols for new coordinates. We will denote partial derivatives by subscripts, for example $F_{z}:=\partial_{z} F$.

Proof of Theorem 2, In what follows, we will use coordinates $(x, y)$ for the twodimensional space of $\beta$-surfaces $U$. We will always work on a single patch of $P S^{\prime}$, with $\lambda$ a standard coordinate on one patch of the $\mathbb{C P}^{1}$ fibre. The projectivization of (4.1) is

$$
\begin{aligned}
& L_{0}=\mathbf{e}_{00^{\prime}}+\lambda \mathbf{e}_{01^{\prime}}+\left(f_{0}+\lambda f_{1}+\lambda^{2} f_{2}+\lambda^{3} f_{3}\right) \partial_{\lambda}, \\
& L_{1}=\mathbf{e}_{01^{\prime}}+\lambda \mathbf{e}_{11^{\prime}}+\left(A_{0}+\lambda A_{1}+\lambda^{2} A_{2}+\lambda^{3} A_{3}\right) \partial_{\lambda},
\end{aligned}
$$

where the $f_{\alpha}$ and $A_{\alpha}$ are functions on $M$ derived from primed connection coefficients.

We can trivialize $P T U$ by first choosing a two dimensional surface in $M$, transverse to the $\beta$-surfaces, and trivializing $P S^{\prime}$ over this, using the standard two patch coordinates for $\mathbb{C P}^{1}$. Then define a trivialization over the rest of $P S^{\prime}$ by requiring constant coordinate on each leaf of $\mathcal{D}$ (this will be a base dependent Möbius transformation of any other trivialization of $P S^{\prime}$ using a standard two patch trivialization of $\mathbb{C P}^{1}$, since any two standard trivializations of $\mathbb{C P}^{1}$ are related by a Möbius transformation). This gives a trivialization $P T U \cong U \times \mathbb{C P}^{1}$. The special feature of this particular trivialization is that $\tilde{K}$ and $W$ will have no vertical terms, because it was defined by saying that the fibre coordinate is constant along them.

We will use the conventions of Lemma 3, that is we choose a tetrad with $K=\mathbf{e}_{00^{\prime}}$, and the tangent planes to the $\beta$-surfaces are spanned by $K$ and $\mathbf{e}_{01^{\prime}}$. Now choose a coordinate system $(t, x, y, z)$ such that $K=\partial_{t}$, and a conformal factor so that $K$ is pure Killing. Any tetrad can then be written in these coordinates without any $t$ dependence. Then $\left[\mathbf{e}_{00^{\prime}}, \mathbf{e}_{01^{\prime}}\right]=0$ and we can in addition choose the $z$ coordinate such that $\mathbf{e}_{01^{\prime}}=\partial_{z}$. Then we have

$$
\begin{aligned}
\tilde{K} & =\partial_{t}, \\
L_{0} & =\partial_{t}+\lambda \partial_{z}+f(x, y, z, \lambda) \partial_{\lambda} .
\end{aligned}
$$

Note that $f$ does not depend on $t$ because it is composed from connection coefficients, which do not depend on $t$ since it does not occur in the metric. Also note that $\tilde{K}=\partial_{t}$ because $L_{0}, L_{1}$ do not contain functions of $t$ so it commutes with both. As vector fields on the base, $\partial_{x}$ and $\partial_{y}$ are transverse to the $\beta$-surfaces so are coordinates on $U$.

Now we will alter the $\lambda$ coordinate, using a trivialization as described above, so that $L_{0}$ has no $\partial_{\lambda}$ terms. This is achieved by a Möbius transformation, $\lambda \rightarrow$ $(\beta+\delta \lambda) /(\alpha+\gamma \lambda)$, where $\alpha, \beta, \gamma, \delta$ are functions on $M$. Now the new $\lambda$ coordinate satisfies $\tilde{K}(\lambda)=L_{0}(\lambda)=0$. Therefore $\alpha, \ldots, \delta$ do not depend on $t$, from the first of 
these. This gives the following general form:

$$
\begin{aligned}
\tilde{K} & =\partial_{t} \\
L_{0} & =\alpha \partial_{t}+\beta \partial_{z}+\lambda\left(\gamma \partial_{t}+\delta \partial_{z}\right)
\end{aligned}
$$

Now from Theorem 11, we know that $L_{1}$ must define a projective structure on $U$, the space of $\beta$-surfaces. In fact this can be seen directly using our coordinate choices ${ }^{2}$. Clearly $U$ has coordinates $(x, y)$, since the $\beta$-surfaces are spanned by $\left(\partial_{t}, \partial_{z}\right)$. Also, $\lambda$ is a fibre coordinate on $P T U$, since $\mathcal{D}$ is defined by constant $\lambda$. Since $\left\{L_{0}, L_{1}\right\}$ is an integrable distribution, one can find a non-zero function $f$ on $P S^{\prime}$ such that $\left[L_{0}, f L_{1}\right] \propto L_{0}$. We may therefore assume we have chosen an $L_{1}$ such that $\left[L_{0}, L_{1}\right] \propto L_{0}$. It follows from (5.4) that the coefficients in front of the $\partial_{x}, \partial_{y}, \partial_{\lambda}$ terms in $L_{1}$ do not depend on $z$. Therefore $L_{1}$ must have the following form:

$$
\begin{aligned}
L_{1}:=J_{0}(x, y) \partial_{x} & +J_{1}(x, y) \partial_{y}+\lambda\left(J_{2}(x, y) \partial_{x}+J_{3}(x, y) \partial_{y}\right) \\
+ & \left(A_{0}(x, y)+\lambda A_{1}(x, y)+\lambda^{2} A_{2}(x, y)+\lambda^{3} A_{3}(x, y)\right) \partial_{\lambda} \\
& +(C(x, y, z)+\lambda D(x, y, z)) \partial_{t}+(E(x, y, z)+\lambda F(x, y, z)) \partial_{z}
\end{aligned}
$$

where $J_{0} J_{3}-J_{1} J_{2} \neq 0$. One now observes that the $\partial_{x}, \partial_{y}, \partial_{\lambda}$ terms precisely correspond to a projective structure spray on $P T U$. Since $\mathcal{D}$ is spanned by $\partial_{t}, \partial_{z}$, the quotient of $L_{1}$ by $\mathcal{D}$ gives a projective structure.

To put the projective structure spray occuring in (5.5) into the more standard form (3.4) (i.e. $J_{0}=J_{3}=1, J_{1}=J_{2}=0$ ) it is necessary to perform a Möbius transformation of $\lambda$ depending on $(x, y)$. Since this does not depend on $t$ or $z$, the general form (5.4) of $L_{0}$ is unchanged by this, and we can assume that the projective structure spray in $L_{1}$ is of the form (3.4), which we shall do from now on.

We have found a general form that any $\left\{\tilde{K}, L_{A}\right\}$ can be put into. For it to give an ASD conformal structure, the $L_{A}$ must commute modulo $L_{A}$. Imposing this gives equations for the unknown functions, which will lead us to the metrics appearing in Theorem 2

First, it is convenient to change coordinates yet again, because together with conformal rescaling we can elimate three of the four functions in $L_{0}$. We may assume $\delta \neq 0$ (if $\delta=0$ then $\beta \neq 0$, in which case perform the coordinate change $\lambda \rightarrow 1 / \lambda$ ).

Now change coordinates by $(t, x, y, z) \rightarrow(t+j(x, y, z), x, y, k(x, y, z))$, where $k_{z} \neq$

\footnotetext{
${ }^{2}$ The following argument does not require analyticity, only smoothness. Consequently Theorem 2 will turn out to be valid for smooth conformal structures, not just analytic ones. The smooth generalisation of Theorem 1 could perhaps be established using techniques introduced in [18].
} 
0. A suitable choice of $j$ and $k$, and conformal rescaling, simplifies $L_{0}$ so that finally

$$
\begin{aligned}
\tilde{K}= & \partial_{t} \\
L_{0}= & \partial_{t}-\beta(x, y, z) \partial_{z}+\lambda \partial_{z}, \\
L_{1}= & \partial_{x}+\lambda \partial_{y}+\left(A_{0}(x, y)+\lambda A_{1}(x, y)+\lambda^{2} A_{2}(x, y)+\lambda^{3} A_{3}(x, y)\right) \partial_{\lambda} \\
& +(C(x, y, z)+\lambda D(x, y, z)) \partial_{t}+(E(x, y, z)+\lambda F(x, y, z)) \partial_{z} .
\end{aligned}
$$

One can read off a metric $g \in[g]$ corresponding to the twistor distribution given by (5.7) and (5.8) by comparing with (5.1) and (5.2) and reading off a null tetrad. One finds that $\mathbb{K} \wedge d \mathbb{K}=\beta_{z} d x \wedge d y \wedge d z$, where $\mathbb{K}=g\left(\partial_{t},.\right)$. Thus the twist of the Killing vector $\partial_{t}$ vanishes iff $\beta$ does not depend on $z$. Since existence of twist is a conformally invariant property, the cases $\beta_{z}=0$ and $\beta_{z} \neq 0$ are genuinely distinct, not an artefact of our coordinate choices. We now analyse each in turn.

Twist-free case: $\beta_{z}=0$. Calculating the commutator of $L_{0}$ and $L_{1}$ we obtain

$$
\begin{aligned}
{\left[L_{0}, L_{1}\right]=\quad } & (-\beta+\lambda)\left(C_{z}+\lambda D_{z}\right) \partial_{t}+\left(\beta_{x}+\lambda \beta_{y}-\beta E_{z}-\lambda \beta F_{z}+\lambda E_{z}+\lambda^{2} F_{z}-\right. \\
& \left.\left(A_{0}+\lambda A_{1}+\lambda^{2} A_{2}+\lambda^{3} A_{3}\right)\right) \partial_{z} .
\end{aligned}
$$

Since we require $\left\{L_{0}, L_{1}\right\}$ to be integrable, this must be a multiple of $L_{0}$. We deduce that ${ }^{3}$

$$
\left[L_{0}, L_{1}\right]=(-\beta+\lambda)\left(C_{z}+\lambda D_{z}\right) L_{0} .
$$

Now comparing the $\partial_{z}$ coefficients of (5.9) and (5.10) we get four equations, one for each power of $\lambda$. We can solve three of them, and use $L_{1} \rightarrow L_{1}-C L_{0}$ which does not change the conformal structure. This yields

$$
\begin{aligned}
& L_{1}=\partial_{x}+\lambda \partial_{y}+\left(A_{0}+\lambda A_{1}+\lambda^{2} A_{2}+\lambda^{3} A_{3}\right) \partial_{\lambda} \\
& \quad+\lambda\left(-z A_{3}+Q\right) \partial_{t}+\left(z\left(-\beta_{y}+A_{1}+\beta A_{2}+\beta^{2} A_{3}\right)++\lambda\left(z\left(A_{2}+2 \beta A_{3}\right)+P\right)\right) \partial_{z},
\end{aligned}
$$

where $P$ and $Q$ are arbitrary functions of $(x, y)$ and we have eliminated one arbitrary function by translating the $z$ coordinate. There is one remaining equation to solve, corresponding to the $\lambda^{0}$ coefficient of $\partial_{z}$. This equation is as follows:

$$
\beta_{x}+\beta \beta_{y}-A_{0}-\beta A_{1}-\beta^{2} A_{2}-\beta^{3} A_{3}=0 .
$$

The metric (1.2) in Theorem 2 corresponds to the twistor distribution given by $L_{0}$, with $\beta_{z}=0$, and (5.11). If $\beta(x, y)$ is regarded as defining a section of PTU, then

\footnotetext{
${ }^{3}$ In 3 the resulting equations are interpreted as a special case of a gauge theory defined on a projective surface. A solution is called a projective Higgs pair. This also applies to the twisting case.
} 
(15.12) says that this section is tangent to lifted geodesics of the projective structure. In terms of the base, a solution is given by a congruence of geodesics.

Twisting case: $\beta_{z} \neq 0$. We may perform a coordinate transformation $z \rightarrow$ $\beta(x, y, z)$. This does not affect the general form (5.8) of $L_{1}$. Performing the coordinate change and dividing by $\beta_{z}$ gives the following form for $L_{0}$ :

$$
L_{0}=H(x, y, z) \partial_{t}-z \partial_{z}+\lambda \partial_{z},
$$

where $H$ is a non-zero arbitrary function. Calculating the commutator gives

$$
\begin{aligned}
{\left[L_{0}, L_{1}\right]=} & \left((-z+\lambda)\left(C_{z}+\lambda D_{z}\right)-(E+\lambda F) H_{z}\right) \partial_{t}+ \\
& \left((-z+\lambda)\left(E_{z}+\lambda F_{z}\right)-(E+\lambda F)-\left(A_{0}+\lambda A_{1}+\lambda^{2} A_{2}+\lambda^{3} A_{3}\right)\right) \partial_{z} .
\end{aligned}
$$

We require $\left[L_{0}, L_{1}\right]=\alpha L_{0}$ for some function $\alpha(x, y, z, \lambda)$, which is at most quadratic in $\lambda$, since otherwise $\alpha L_{0}$ will contain powers of $\lambda$ greater than three, and such terms do not occur in the commutator above. We make a replacement $L_{1} \rightarrow L_{1}-F L_{0}$, and analyze equations obtained from comparing coefficients of $\partial_{z}, \partial_{t}$. This puts $L_{1}$ in the form

$$
\begin{aligned}
L_{1}= & \partial_{x}+\lambda \partial_{y}+\left(A_{0}+\lambda A_{1}+\lambda^{2} A_{2}+\lambda^{3} A_{3}\right) \partial_{\lambda} \\
& +(C+\lambda D) \partial_{t}+\left(A_{0}+z A_{1}+z^{2} A_{2}+z^{3} A_{3}\right) \partial_{z},
\end{aligned}
$$

where $C(x, y, z), D(x, y, z), H(x, y, z)$ satisfy

$$
\begin{aligned}
C_{z}-2 z D_{z} & =-H A_{2}+H_{y} . \\
D_{z} & =-H A_{3} .
\end{aligned}
$$

and

$$
\left(\partial_{x}+z \partial_{y}+\left(A_{0}+z A_{1}+z^{2} A_{2}+z^{3} A_{3}\right) \partial_{z}\right) H=0 .
$$

The only things remaining now are to find expressions for $C$ and $D$ and construct the metric. In order to integrate equations (5.14) it is convenient to express $H(x, y, z)$ as the second derivative of another function $G(x, y, z)$, i.e. we set

$$
H(x, y, z)=\frac{\partial^{2} G}{\partial z^{2}}(x, y, z)
$$

Then equations (5.14) integrate to give

$$
\begin{aligned}
C & =-G_{z} A_{2}-2 A_{3}\left(z G_{z}-G\right)+G_{z y}+\rho(x, y), \\
D & =-G_{z} A_{3}+\sigma(x, y),
\end{aligned}
$$


where $\rho$ and $\sigma$ are arbitrary functions. Notice that $G$ has a 'gauge freedom' $G \rightarrow$ $G+z \gamma(x, y)+\delta(x, y)$, since (1.4) will still be satisfied. Using this and a coordinate change $t \rightarrow t+\xi(x, y)$, one can set the functions $\rho$ and $\sigma$ to zero. The twistor distribution $\left\{L_{0}, L_{1}\right\}$ is now fully determined:

$$
\begin{aligned}
L_{0}= & G_{z z} \partial_{t}-z \partial_{z}+\lambda \partial_{z} \\
L_{1}= & \partial_{x}+\lambda \partial_{y}+\left(A_{0}+\lambda A_{1}+\lambda^{2} A_{2}+\lambda^{3} A_{3}\right) \partial_{\lambda} \\
& \left.+\left(-G_{z} A_{2}-2 A_{3}\left(z G_{z}-G\right)+G_{z y}\right)-\lambda\left(G_{z} A_{3}\right)\right) \partial_{t} \\
& +\left(A_{0}+z A_{1}+z^{2} A_{2}+z^{3} A_{3}\right) \partial_{z} .
\end{aligned}
$$

The distribution is integrable iff $G$ satisfies (1.4). Calculating the corresponding null tetrad gives the conformal structure (1.3) in Theorem 2 , $\square$

\section{$6 \quad$ Examples}

\subsection{Neutral Fefferman conformal metrics.}

If $G_{z z}$ is simply a constant, then (1.4) is satisfied. So given any projective structure and setting $G_{z z}=1$ we obtain a family of conformal structures with twist which reduce to the given projective structure. Solving for $G$ gives

$$
G=\frac{z^{2}}{2}+z \gamma(x, y)+\delta(x, y)
$$

The corresponding metric takes the form

$$
\begin{array}{r}
\left(d t+\left((z+\gamma) A_{3}+\sigma\right) d y+\left((z+\gamma) A_{2}+2 A_{3}\left(\frac{z^{2}}{2}-\delta\right)-\gamma_{y}+\rho\right) d x\right)(d y-z d x) \\
-\left(d z-\left(A_{0}+z A_{1}+z^{2} A_{2}+z^{3} A_{3}\right) d x\right) d x
\end{array}
$$

where we have chosen not to eliminate $\sigma$ and $\rho$. By direct calculation one can show that the ASD Weyl tensor has Petrov-Penrose type III or N, and it is type N precisely when the following hold:

$$
\begin{aligned}
\gamma A_{3}+\sigma & =\frac{1}{3} A_{2}, \\
\gamma A_{2}-2 A_{3} \delta-\gamma_{y}+\rho & =\frac{2}{3} A_{1} .
\end{aligned}
$$

One can always choose $\rho, \sigma, \gamma, \delta$ so that these are satisfied. In this case, the metric is the same as (31) in [20], with their $Q$ cubic in $p$. These are neutral signature analogues of Fefferman metrics. 


\subsection{ASD pp-waves}

Notice that the metric (1.2) does not explicitly contain the function $A_{0}(x, y)$ of the projective structure. The metric is always ASD for any choice of $\beta, A_{1}, A_{2}, A_{3}$; one can regard (5.12) as giving $A_{0}(x, y)$ in terms of these functions. One the other hand, if one wants to specify $A_{0}$, then one must choose a solution of (5.12) for $\beta$. In the special case $A_{0}=0$, we have the solution $\beta=0$. One then obtains the following metric:

$$
g=\left(d t+\left(P+z A_{2}\right) d x+\left(Q+z A_{3} d y\right)\right) d y-\left(d z+z A_{1} d x\right) d x
$$

Different choices of function $\beta(x, y)$ in (1.2) can give rise to different metrics. Suppose we choose the flat projective structure. Then $\beta(x, y)$ must satisfy the equation (1.6) with $A_{\alpha}=0$. By direct calculation one can show that the metric (1.2) is type III iff $\beta_{y y} \neq 0$, otherwise it is type $\mathrm{N}$. So the conformal structures with $\beta_{y y}=0$ and $\beta_{y y} \neq 0$ are genuinely distinct.

\subsection{Pseudo-hyper-Kähler metrics}

We will find some examples of neutral ASD metrics with null conformal Killing vectors by independent means, and interpret them using our results. We will use Plebański's method [23] adapted to neutral signature, which converts the problem of finding Ricci-flat ASD neutral metrics, or pseudo-hyper-Kähler, to the problem of solving a non-linear second order PDE. He showed that such metrics are locally of the form

$$
g=d Y\left(d T-\Theta_{X X} d Y-\Theta_{T X} d Z\right)-d Z\left(d X+\Theta_{T T} d Z+\Theta_{T X} d Y\right)
$$

where $\Theta(T, X, Y, Z)$ satisfies the 'second Heavenly Equation':

$$
\Theta_{Y T}-\Theta_{Z X}+\Theta_{T T} \Theta_{X X}-\Theta_{X T}^{2}=0
$$

The primed connection coefficients vanish when using the tetrad indicated in (6.19), so there is a basis of covariantly constant primed spinors $o^{A^{\prime}}=(1,0), \iota^{A^{\prime}}=(0,-1)$. There is therefore also a basis $\Sigma^{A^{\prime} B^{\prime}}$ of covariantly constant null self-dual two forms, written in spinors as follows:

$$
\begin{aligned}
\Sigma^{0^{\prime} 0^{\prime}} & =\frac{1}{2} \iota_{A^{\prime} \iota_{B^{\prime}}} \epsilon_{A B} \theta^{A A^{\prime}} \wedge \theta^{B B^{\prime}} \\
\Sigma^{0^{\prime} 1^{\prime}}=\Sigma^{1^{\prime} 0^{\prime}} & =\frac{1}{2} o_{\left(A^{\prime} \iota_{B^{\prime}}\right.} \epsilon_{A B} \theta^{A A^{\prime}} \wedge \theta^{B B^{\prime}} \\
\Sigma^{1^{\prime} 1^{\prime}} & =\frac{1}{2} o_{A^{\prime} O_{B^{\prime}}} \epsilon_{A B} \quad \theta^{A A^{\prime}} \wedge \theta^{B B^{\prime}} .
\end{aligned}
$$


Using the identification between two-forms and endomorphisms given by $g$, we can write

$$
R=\Sigma^{0^{\prime} 0^{\prime}}-\Sigma^{1^{\prime} 1^{\prime}}, \quad I=\Sigma^{0^{\prime} 0}+\Sigma^{1^{\prime} 1}, \quad S=\Sigma^{0^{\prime} 1^{\prime}} .
$$

As endomorphisms, these satisfy

$$
-I^{2}=R^{2}=S^{2}=\mathrm{Id}, \quad I R S=\mathrm{Id},
$$

which is easy to check using their spinor forms. There is a hyperboloid's worth of almost complex structures, $a I+b R+c S$, where $a^{2}-b^{2}-c^{2}=1$, which are parallel and hence integrable. This is a pseudo-hyper-Kähler structure.

Now writing (A.2) using spinors by means of (2.4) and (A.1) gives

$$
\iota^{A} O^{A^{\prime}} C_{A B C D} \epsilon_{A^{\prime} B^{\prime}} \epsilon_{C^{\prime} D^{\prime}}=\nabla_{B B^{\prime}}\left(\phi_{C^{\prime} D^{\prime}} \epsilon_{C D}+\psi_{C D} \epsilon_{C^{\prime} D^{\prime}}+\frac{1}{2} \eta \epsilon_{C D} \epsilon_{C^{\prime} D^{\prime}}\right),
$$

where we have used Ricci flatness and anti-self-duality. For a pure Killing vector or a homothety ( $\eta$ constant), it follows that

$$
\nabla_{A A^{\prime}} \phi_{B^{\prime} C^{\prime}}=0
$$

Therefore $\phi_{B^{\prime} C^{\prime}}$ is actually constant in the basis shown in (6.19). Now let us suppose we have a null Killing vector which preserves the $\alpha$-plane distribution spanned by $O^{A^{\prime}} \mathbf{e}_{A A^{\prime}}$. Then $K=\iota^{A} O^{A^{\prime}} \mathbf{e}_{A A^{\prime}}$, for some $\iota^{A}$ and using (2.6) and (6.25) we get

$$
\phi_{B^{\prime} C^{\prime}}=a_{1} o_{B^{\prime}} O_{C^{\prime}}+a_{2} o_{\left(B^{\prime} \iota_{\left.C^{\prime}\right)}\right.},
$$

for constant $a_{1}, a_{2}$. Consider the three distinct cases: $\phi_{B^{\prime} C^{\prime}}$ vanishing $\left(a_{1}=a_{2}=0\right)$, non-vanishing but degenerate $\left(a_{1} \neq 0, a_{2}=0\right)$, and non-degenerate $\left(a_{1}=0, a_{2} \neq 0\right)$. For $K=\partial_{T}$ we get the first case, $K=Y \partial_{X}+Z \partial_{T}$ the second, and $T \partial_{T}+X \partial_{X}$ the third, and with some efford it can be shown that these choices are canonical ( the first two cases were analysed in [8]). In order for any of these to be Killing, an equation for $\Theta$ coming from the Killing equation must be satisfied. In fact we were only able to fully solve for the first two cases.

- $K=\partial_{T}$

Since $\partial_{T}$ has no twist we expect this to be of the form (1.2). It is a neutral signature version of a tri-holomorphic Killing vector; i.e. it Lie-derives $I, R, S$. Solving the Killing equations in conjunction with (6.20) results in the following metric:

$$
g=d Y d T-d Z d X-Q(X, Y) d Y^{2}
$$

where $Q$ is an arbitrary function. This is simply the split-signature pp-wave metric, and is a special case of (6.18). Here $K$ is a self-dual Killing vector in the sense of Gibbons et al. [1]. 
The local expression (6.26) in this example corresponds to a class of global neutral metrics on compact four-manifolds. To see this we compactify the flat projective space $\mathbb{R}^{2}$ to two-dimensional torus $U=T^{2}$ with the projective structure coming from the flat metric. Both $T$ and $Z$ in (6.26) are taken to be periodic, thus leading to $\hat{\pi}: M \longrightarrow U$, the holomorphic toric fibration over a torus. Assume the suitable periodicity on the function $Q: U \longrightarrow \mathbb{R}$. This leads to a commutative diagram

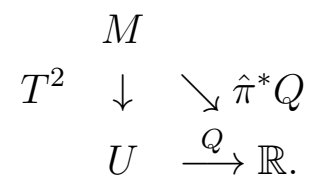

This example can be put into the framework of [15] and [9], where $M$ is regarded as a primary Kodaira surface $\mathbb{C}^{2} / G$ and $G$ is the fundamental group of $M$ represented injectively in the group of complex affine transformations of $\mathbb{C}^{2}$. In this framework the Kähler structure on $M$ is given by $\Omega_{f l a t}+i \partial \bar{\partial}\left(\hat{\pi}^{*} Q\right)$, where $\left(\partial, \Omega_{f l a t}\right)$ is the flat Kähler structure on the Kodaira surface induced from $\mathbb{C}^{2}$.

- $K=Y \partial_{X}+Z \partial_{T}$

Again, this is twist-free and we expect the metric to be of the form (1.2). Solving the Killing equations in conjunction with (6.20) results in the following metric:

$$
g=d Y d T-d Z d X-\frac{H\left(\frac{Y}{Y T-Z X}, \frac{Z}{Y T-Z X}\right)}{(Y T-Z X)^{3}}(Y d Z-Z d Y)^{2},
$$

where $H$ is an arbitrary analytic function of two variables. This is a generalization of the Sparling-Tod metric [24]. It is easy to show that the arguments of $H$ are in fact constant on the special $\beta$-surfaces, so serve as coordinates on $U$.

Using the following coordinate transformation

$$
\begin{aligned}
t & =-\frac{1}{2}\left(\frac{X}{Y}+\frac{T}{Z}\right), \\
z & =(Y Z)^{-\frac{1}{2}} \\
x & =\frac{Y T-X Z}{(Y Z)^{\frac{1}{2}}} \\
y & =\log \left(\frac{Z}{Y}\right) .
\end{aligned}
$$

the metric (6.27) takes the following form:

$$
g=\frac{1}{z^{2}}\left(d y d t-d z d x+z A_{3}(x, y) d y^{2}\right),
$$


where now the Killing vector is $\partial_{t}$. Multiplying by the conformal factor $z^{2}$, we get a special case of (6.18). The projective structure is non-trivial, unlike for the pp-wave above. The projective structure is special in that it depends on only one arbitrary function.

- $T \partial_{T}+X \partial_{X}$

In this case we were not able to fully solve the Killing equations in conjunction with (6.20). This Killing vector is twisting, so the answer must be of the form (1.3).

\subsection{Pseudo-hyper-hermitian conformal structures}

This is a generalization of the pseudo-hyper-Kähler case discussed in the last section. We will refer to a neutral metric $g$ as pseudo-hyper-hermitian (also called hyperpara-hermitian [12]) when there exist endomorphisms $I, R, S$ satisfying the algebra (6.24), such that any complex structure $\mathcal{J}_{(a, b, c)}=a I+b R+c S$ is integrable for $a^{2}-b^{2}-c^{2}=1$, and $g$ is hermitian with respect to any of these complex structures. For $g$ to be hermitian with respect to a complex structure $\mathcal{J}$ means $g(\mathcal{J} X, \mathcal{J} Y)=$ $g(X, Y)$. Note that for pseudo-hyper-Kähler, the endomorphisms $I, R, S$ must also be covariantly constant with respect to the Levi-Civita connection of $g$.

In [5], it is shown that one can always find a tetrad for a pseudo-hyper-hermitian metric such that the twistor distribution has no $\partial_{\lambda}$ terms. Equivalently, the twistor space fibres over $\mathbb{C P}^{1}$. Now let us suppose that we have a null conformal Killing that is tri-holomorphic, i.e. it preserves $I, R$ and $S$ and so it preserves the holomorphic fibration $\mathcal{P} \mathcal{T} \rightarrow \mathbb{C P}^{1}$. All such cases are classified by the following

Proposition 2. All pseudo-hyper-hermitian ASD metrics with triholomorphic null conformal Killing vectors are of the form (1.2) or (1.3) up to a conformal factor, where the corresponding ODE (1.5) is point equivalent to a derivative of a first order $O D E$.

Proof. Let $g$ be a pseudo-hyper-hermitian ASD metric, and $K$ be a triholomorphic conformal Killing vector. Since $g$ is ASD, it follows from Theorem 2 that there are coordinates such that, up to a conformal factor, $g$ is of the form (1.2) or (1.3). From [5], it is possible to find a tetrad such that the twistor distribution has no $\partial_{\lambda}$ terms. Now a change in tetrad corresponds to a Möbius transformation of $\lambda$. Since $K$ is triholomorphic, its lift will have no $\partial_{\lambda}$ terms in the tetrad where the twistor distribution has no $\partial_{\lambda}$ terms. Therefore the Möbius transform does not depend on $t$, otherwise $\partial_{t}$ will no longer Lie-derive the twistor distribution (one would have to add $\partial_{\lambda}$ terms). Furthermore, the Möbius transformation does not depend on $z$, otherwise $\partial_{\lambda}$ terms will be introduced into $L_{0}$. Hence there is a Möbius transformation of $\lambda$, depending only on $(x, y)$, such that the $\partial_{\lambda}$ terms in $L_{1}$ are eliminated. 
After this change in $\lambda$, the projective structure spray in $L_{1}$ will be of the following form:

$$
\Theta=a \partial_{x}+b \partial_{y}+\lambda\left(c \partial_{x}+e \partial_{y}\right)
$$

where $a, b, c, e$ are functions of $(x, y)$ with $a e-b c \neq 0$. Coordinate freedom $(x, y) \rightarrow$ $(\hat{x}(x, y), \hat{y}(x, y))$ and scaling freedom (the projective structure is unchanged if $\Theta$ is multiplied by a non-zero function) allows us to set $a=1, c=0, e=1$, giving $\Theta=\partial_{x}+(b+\lambda) \partial_{y}$. Now perform another Möbius transformation $\lambda \rightarrow b+\lambda$, which gives the following spray:

$$
\partial_{x}+\lambda \partial_{y}+\left(b_{x}+\lambda b_{y}\right) \partial_{\lambda}
$$

This corresponds to the second-order ODE

$$
\frac{d^{2} y}{d x^{2}}=A_{1}(x, y)\left(\frac{d y}{d x}\right)+A_{0}(x, y)
$$

where $A_{1}=\frac{\partial b}{\partial y}, A_{0}=\frac{\partial b}{\partial x}$ for a function $b(x, y)$. This is the derivative of the general first-order ODE

$$
\frac{d y}{d x}=b(x, y)
$$

Hence the original projective structure is point-equivalent to the one corresponding to (6.29).

Note that if a (holomorphic) projective structure spray contains no $\partial_{\lambda}$ terms, its twistor space fibres over $\mathbb{C P}^{1}$, since each integral curve can be labelled by the $\lambda$ coordinate. So a by-product of the proof of the above Proposition and Theorem 3$]$ is the following

Proposition 3. There is a one to one correspondence between holomorphic 2D projective structures s.t. the corresponding second order ODE is point equivalent to the derivative of a first order ODE, and complex surfaces which contain a holomorphic curve with normal bundle $\mathcal{O}(1)$ and fiber holomorphically over $\mathbb{C P}^{1}$.

This is of interest purely as a statement about projective structures. Note that although all first order ODEs can be transformed to the trivial first order ODE $d y / d x=0$ by coordinate transformation, this does not mean that the derivative of any such equation is flat, in the sense of Section 3.2. This can be shown by calculating the invariant (3.7) for (6.29) and showing that it does not necessarily vanish.

\subsection{Conformal structures containing no Ricci-flat metrics}

In this section we show that there are conformal structures of the form (1.2) which do not contain Ricci-flat metrics. Before doing so we discuss the Petrov-Penrose classification for the conformal structures (1.2) and (1.3). 
Proposition 4. Let $K^{A A^{\prime}}=\iota^{A} O^{A^{\prime}}$ be a null conformal Killing vector for ASD conformal structure. Then $\iota^{A}$ is a principal direction, that is

$$
\iota^{A} \iota^{B} \iota^{C} \iota^{D} C_{A B C D}=0 .
$$

Moreover if the twist of $K$ vanishes the conformal structure is of type III or $N$, that is

$$
\iota^{A} \iota^{B} C_{A B C D}=0
$$

Proof. From (2.5) we have

$$
\nabla_{A A^{\prime}}\left(\iota^{C} \iota^{D} \psi_{C D}\right)=0
$$

Expanding this out we obtain

$$
\iota^{B} \iota^{C} \nabla_{A A^{\prime}} \psi_{B C}=-2 \psi_{B C} \iota^{C} \nabla_{A A^{\prime}} \iota^{B}=\iota_{A} \mu_{A^{\prime}},
$$

for some spinor $\mu_{A^{\prime}}$. The last equality follows from (2.5) and (2.7).

Now pick a conformal frame in which $K$ is a pure Killing vector. The well known identity $\nabla_{a} \nabla_{b} K_{c}=R_{b c a d} K^{d}$ implies

$$
\nabla_{A}^{A^{\prime}} \psi_{B C}=-2 C_{A B C}^{D} K_{D}^{A^{\prime}}-2 K_{(A}^{B^{\prime}} \Phi_{B C) B^{\prime}}^{A^{\prime}}+\frac{1}{6} R \epsilon_{A(B} K_{C)}^{A^{\prime}}-\frac{4}{3} \epsilon_{A(B} \Phi_{C)}^{D D^{\prime} A^{\prime}} K_{D D^{\prime}}
$$

On contracting both sides by $\iota^{A} \iota^{B} \iota^{C}$ and using (6.33), all terms vanish except the term involving $C_{A B C}^{D}$, giving (6.31).

Now let us assume that $K$ is non-twisting, i. e. $\mathbb{K} \wedge d \mathbb{K}=0$ where $\mathbb{K}:=g(K$,$) . The$ Frobenius theorem implies the existence of functions $P$ and $Q$ such that $\mathbb{K}=P d Q$. We can now choose a conformal factor such that $d \mathbb{K}=0$. Then $K$ is covariantly constant $\left(\nabla_{a} K_{b}=0\right)$, and we deduce

$$
\begin{aligned}
\nabla_{A A^{\prime}} \iota_{B} & =A_{A A^{\prime} \iota_{B}}, \\
\nabla_{A A^{\prime}} O_{B^{\prime}} & =-A_{A A^{\prime}} O_{B^{\prime}}
\end{aligned}
$$

for some one-form $A_{A A^{\prime}}$. Consider the spinor Ricci identity [22]

$$
\triangle_{A^{\prime} B^{\prime}} O_{C^{\prime}}=\left(C_{A^{\prime} B^{\prime} C^{\prime} D^{\prime}}-\frac{1}{12} R \epsilon_{D^{\prime}\left(A^{\prime}\right.} \epsilon_{\left.B^{\prime}\right) C^{\prime}}\right) o^{D^{\prime}}
$$

where $\triangle_{A^{\prime} B^{\prime}}=\nabla_{A\left(A^{\prime}\right.} \nabla_{\left.B^{\prime}\right)}^{A}$. Substituting (6.35) into this and using $C_{A^{\prime} B^{\prime} C^{\prime} D^{\prime}}=0$ gives

$$
o_{C^{\prime}} \nabla_{A\left(A^{\prime}\right.} A_{\left.B^{\prime}\right)}^{A}=-\frac{1}{12} R o_{\left(A^{\prime}\right.} \epsilon_{\left.B^{\prime}\right) C^{\prime}}
$$


By contracting with $o^{C^{\prime}}$ we find $R=0$. Now consider the Ricci identity

$$
\triangle_{A B} \iota_{C}=\left(C_{A B C D}-\frac{1}{12} R \epsilon_{D(A} \epsilon_{B) C}\right) \iota^{D} .
$$

Substituting $R=0$ and (6.34) into this gives

$$
\iota_{C} \nabla_{A^{\prime}(A} A_{B)}^{A^{\prime}}=C_{A B C D} \iota^{D}
$$

Contracting this with $\iota^{C}$ gives (6.32), from which it follows that the curvature is type III or N.

In the twisting case the algebraic type of the Weyl spinor can be general. This can be shown by using the following two scalar invariants [22]:

$$
I=C_{A B C D} C^{A B C D}, \quad J=C_{A B}^{C D} C_{C D} \stackrel{E F}{C_{E F}^{A B}} .
$$

The condition for type III is $I=J=0$, and for type II that $I^{3}=6 J^{2}$. Now consider the metric (1.3), with the flat projective structure $A_{i}=0, i=0, \ldots, 3$. The function $G_{z z}$ satisfies

$$
\left(\partial_{x}+z \partial_{y}\right) G_{z z}=0
$$

which is solved in general when $G_{z z}$ is an arbitrary function of $(z x-y)$. Suppose $G$ is given by:

$$
G(x, y, z)=\frac{\mathbf{e}^{z x-y}}{x^{2}}+z B(x, y)
$$

where $B(x, y)$ is arbitrary, so $G_{z z}=\mathbf{e}^{z x-y}$. Then the two scalar invariants are as follows:

$$
\begin{aligned}
I & =-\frac{3}{2} x B_{y y} \mathbf{e}^{-3(z x-y)}, \\
J & =\frac{3}{8} x\left(x B_{y y x}+3 B_{y y}+x z B_{y y y}\right) \mathbf{e}^{-4(z x-y)} .
\end{aligned}
$$

Therefore, from the conditions above, the metric is neither type II nor type III.

To find metrics that are not conformally Ricci-flat we use results of Szekeres [25]. Although these were derived for Lorentzian signature, they can also be applied to our ASD neutral signature case, essentially because the Weyl curvature is still made up of a single spinor $C_{a b c d}=C_{A B C D} \epsilon_{A^{\prime} B^{\prime}} \epsilon_{C^{\prime} D^{\prime}}$ as in the Lorentzian case (of course in Lorentzian case it is complex hermitian, not real).

Consider the metric (6.18) with $A_{1}=0$. By direct calculation, one finds that $C_{A B C D}$ is type $\mathrm{N}$ iff $\left(A_{2}\right)_{x}=0$, otherwise it is type III. Now suppose (1.2) is type III, i.e. $\left(A_{2}\right)_{x} \neq 0$. The reason for this is that we can apply a result of Szekeres to obtain 
an obstruction to Ricci-flatness. It is shown in [25] that for types I, II, D or III, a necessary condition for existence of a Ricci-flat metric in the conformal class is the following tensor equation

$$
-\frac{1}{2} C_{p q f h} C_{r s}{ }^{f h} C_{a b c ; d}{ }^{d}+\left(C_{p q}^{d f} C_{r s f ; h}^{h}+C_{r s}^{d f} C_{p q f ; h}^{h}\right)=0 .
$$

This is just the tensor version of the spinor identity (3.1), page 209 25]. Calculating this one finds that $\left(A_{2}\right)_{x x}$ is an obstruction to its vanishing (we used MAPLE for the calculation). Therefore we have a class of non-conformally vacuum type III neutral ASD conformal structures with non-twisting null conformal Killing vectors.

\section{Twistor reconstruction}

We have shown that when a conformal structure $[g]$ has a null conformal Killing vector, the twistor space $\mathcal{P} \mathcal{T}$ fibres over the twistor space of a projective structure, and we have classified the possible local forms for such conformal structures.

The twistor lines in a projective structure twistor space $Z$ have normal bundle $\mathcal{O}(1)$. The twistor lines in a conformal structure twistor space have normal bundle $\mathcal{O}(1) \oplus \mathcal{O}(1)$. Let $\mathcal{B}$ be a holomorphic fibre bundle over $Z$ with one dimensional fibres. Let $\hat{u}$ be a twistor line in $Z$. Then if we want $\mathcal{B}$ to be a conformal structure twistor space, the normal bundle of $\hat{u}$ in $\left.\mathcal{B}\right|_{\hat{u}}$ must be $\mathcal{O}(1)$. Given a projective structure twistor space, one way of forming a fibre bundle with the correct property is to take a power of the canonical bundle $\kappa$, which reduces to $\mathcal{O}(-3)$ on twistor lines. The bundle $\kappa^{-1 / 3}$ reduces to $\mathcal{O}(1)$ on twistor lines, and exists provided we take $Z$ to be a suitably small neighbourhood of a twistor line. So the total space of $\kappa^{-1 / 3}$ is a conformal structure twistor space.

Consider the simplest possible case, where $Z$ is the total space of $\mathcal{O}(1)$, corresponding to a flat projective structure. In this case $\kappa^{-1 / 3}$ is the total space of $\mathcal{O}(1) \oplus \mathcal{O}(1)$, the twistor space of the flat conformal structure. To go further, note that given a line bundle $\mathcal{A}$ over $Z$ which reduces to $\mathcal{O}(1)$ on twistor lines, any affine bundle modelled on $\mathcal{A}$ will also have the correct property on twistor lines. In the simplest case described above, taking affine bundles modelled on $\kappa^{-1 / 3}$ results in the the twistor space of the pp-wave metric (6.26). In fact, this is precisely the first case discussed by Ward in 28, although he does not phrase it in this way. We will now show how this works.

\subsection{Example 1. PP-waves.}

First we will give a twistorial demonstration of a fact shown in Section 6.3, namely that for a pseudo-hyper-Kähler metric with triholomorphic null Killing vector $K=$ 
$\iota^{A} O^{A^{\prime}} \mathbf{e}_{A A^{\prime}}$ with $O^{A^{\prime}}$ covariantly constant, the resulting projective structure is flat. The twistor space of an analytic pseudo-hyper-Kähler metric fibres over $\mathbb{C P}^{1}, \sigma: \mathcal{P} \mathcal{T} \rightarrow$ $\mathbb{C P}^{1}$ [21, 11]. There is a section $\varpi$ of $\Lambda^{2} \mathcal{P} \mathcal{T} \times \sigma^{*} \mathcal{O}(2)$. This is a symplectic form of 'degree 2 ' on the fibres. In the spin bundle picture, $\varpi$ is the push forward to $\mathcal{P} \mathcal{T}$ of the symplectic form $\Sigma=\Sigma^{A^{\prime} B^{\prime}} \pi_{A^{\prime}} \pi_{B^{\prime}}$ on $S^{\prime}$, where $\boldsymbol{\Sigma}^{A^{\prime} B^{\prime}}$ are defined as in Section 6.3. This form is Lie-derived over the twistor distribution as a consequence of the $\Sigma^{A^{\prime} B^{\prime}}$ being covariantly constant, and is homogeneous in the $\pi_{A^{\prime}}$, so the push-forward is well defined.

As explained in Section 4, $\mathcal{K}$ vanishes on a hypersurface $\mathcal{H}$ in $\mathcal{P} \mathcal{T}$, where $\mathcal{H}$ is the projection to $\mathcal{P} \mathcal{T}$ of the hypersurface $\pi . o=0$ in $S^{\prime}$. For $o^{A^{\prime}}$ covariantly constant, the function $1 /(\pi . o)$ on $S^{\prime}$ gives a section $\zeta$ of $\sigma^{*} \mathcal{O}(-1)$ on $\mathcal{P} \mathcal{T}$, which blows up on $\mathcal{H}$. Then $\zeta \otimes \mathcal{K}$ is a non-vanishing ' $\sigma^{*} \mathcal{O}(1)$-valued' vector field. Now in a local trivialization, $\zeta \otimes \mathcal{K}$ Lie derives the symplectic form $\varpi$, so it is Hamiltonian,

$$
\zeta \otimes \mathcal{K}=\frac{\partial h}{\partial \omega^{A}} \frac{\partial}{\partial \omega_{A}}
$$

where $\varpi=\omega^{0} \wedge \omega^{1}$. Now the $\omega^{A}$ should be regarded as coordinates of 'degree 1 ', that is they are coordinate functions multiplied by a section of $\sigma^{*} \mathcal{O}(1)$. Therefore for the weights to agree, $h$ must be a section of $\kappa^{*} \mathcal{O}(1)$, rather than a bona fide function. This gives a projection $\mathcal{P} \mathcal{T} \rightarrow Z=\mathcal{O}(1)$, with fibres the trajectories of $\zeta \otimes \mathcal{K}$, so the projective structure twistor space is the total space of $\mathcal{O}(1)$, which corresponds to the flat projective structure.

Now suppose we start with the total space of $\mathcal{O}(1)$ as the minitwistor space $Z$. The twistor lines are global holomorphic sections of $\mathcal{O}(1) \rightarrow \mathbb{C P}^{1}$.

We will use a homogeneous coordinate description of $Z=\mathcal{O}(1)$. Let $\pi_{A^{\prime}}$ be homogeneous coordinates for the base $\mathbb{C P}^{1}$ of $Z=\mathcal{O}(1)$, and let $\omega$ be a homogeneous coordinate for the fibre of $Z=\mathcal{O}(1)$. That is, $\mathcal{O}(1)=\left\{\left[\pi_{0^{\prime}}, \pi_{1^{\prime}}, \omega\right]:\left[c \pi_{0^{\prime}}, c \pi_{1^{\prime}}, c \omega\right], c \in\right.$ $\left.\mathbb{C}^{*},\left[\pi_{0^{\prime}}, \pi_{1^{\prime}}\right] \neq[0,0]\right\}$.

Now cover the base $\mathbb{C} \mathbb{P}^{1}$ in $\mathcal{P} \mathcal{T}$ with two open sets $\left(\mathcal{U}_{0}, \mathcal{U}_{1}\right)$, and lift this covering to $\mathcal{P} \mathcal{T}$. Use homogeneous coordinates $\left(\pi_{A^{\prime}}, \omega, \zeta_{i}\right)$ on $\mathcal{U}_{i}$.

The flat twistor space $\mathcal{O}(1) \oplus \mathcal{O}(1)$ can be formed as follows. Consider the projection $\tau: \mathcal{O}(1) \rightarrow \mathbb{C P}^{1}$. Then $\mathcal{O}(1) \oplus \mathcal{O}(1)$ is the pull-back bundle $\tau^{*} \mathcal{O}(1)$ over the total space of $\mathcal{O}(1)$. It is easy to check that this is the same as taking $\kappa^{-1 / 3}$ where $\kappa$ is the canonical bundle of $Z=\mathcal{O}(1)$. To obtain curved twistor spaces, we can take affine bundles over $\mathcal{O}(1)$ modelled on $\tau^{*} \mathcal{O}(1)$. To form these we use the following transition functions:

$$
\zeta_{0}=\zeta_{1}+f\left(\pi_{A^{\prime}}, \omega\right),
$$

where $f \in[f] \in H^{1}\left(Z, \tau^{*} \mathcal{O}(1)\right)$, where $Z$ is $\mathcal{O}(1)$. The cohomology elements $f$ classify affine bundles over $Z$ modelled on $\tau^{*} \mathcal{O}(1)$. 
Global holomorphic sections of $Z \rightarrow \mathbb{C P}^{1}$ are defined by $\omega=P\left(\pi_{A^{\prime}}\right)=\pi_{A^{\prime}} x^{A^{\prime}}$, with $x^{A^{\prime}}=(X, Y)$ say.

The sections of $\mathcal{P} \mathcal{T} \rightarrow \mathbb{C P}^{1}$ are constructed by putting $\zeta_{i}=\pi_{A^{\prime}} t^{A^{\prime}}+f_{i}$, where $t^{A^{\prime}}=-(T, Z)$ say, and $f=f_{0}-f_{1}$. The reason $f$ can be split in this way is that when restricted to a twistor line in $Z, f$ becomes an element of $H^{1}\left(\mathbb{C P}^{1}, \mathcal{O}(1)\right)$, and this group vanishes. To realise a splitting of $f$ we divide it by $\left(\pi_{A^{\prime}} O^{A^{\prime}}\right)^{2}$ for some constant $O^{A^{\prime}}$, to get an element of $H^{1}\left(Z, \tau^{*} \mathcal{O}(-1)\right)$. Then we can use the fact that $H^{0}\left(\mathbb{C P}^{1}, \mathcal{O}(-1)\right)=H^{1}\left(\mathbb{C P}^{1}, \mathcal{O}(-1)\right)=0$, so any element can be written as a difference of coboundaries, and the splitting is unique. These sections are the $\mathbb{C P}^{1}$ twistor lines in $\mathcal{P} \mathcal{T}$; we will refer to these as $\hat{x}$, where $x$ is the point in $M$ with coordinates $\left(t^{A^{\prime}}, x^{A^{\prime}}\right)$.

Let $\rho_{A^{\prime}}$ be homogeneous coordinates on $\mathbb{C P}^{1}$. The splitting is given by the Sparling formula:

$$
\frac{f(\pi, P)}{(\pi . o)^{2}}=\oint_{\Gamma_{0}} \frac{f(\rho, P)}{(\rho . o)^{2} \pi . \rho} \rho . d \rho-\oint_{\Gamma_{1}} \frac{f(\rho, P)}{(\rho . o)^{2} \pi . \rho} \rho d \rho,
$$

where we are using Cauchy's integral formula, and $\Gamma_{i} \subset \hat{x} \cong \mathbb{C P}^{1}$ are contours that bound a region containing the point $\rho_{A^{\prime}}=\pi_{A^{\prime}}$. The measure $\rho . d \rho$ means $\epsilon_{A^{\prime} B^{\prime}} \rho^{A^{\prime}} d \rho^{B^{\prime}}$.

Therefore

$$
f_{i}=\oint_{\Gamma_{i}} \frac{(\pi . o)^{2}}{(\rho . o)^{2}} \frac{f(\rho, P)}{\pi \cdot \rho} \rho . d \rho .
$$

The symplectic form $\varpi$ discussed above is given by by $\varpi=d \omega \wedge d \zeta_{i}$ on $\mathcal{U}_{i}$. Restricting $\varpi$ to a section and taking exterior derivatives keeping $\pi_{A^{\prime}}$ constant, we obtain a formula for $\Sigma$, the pull-back of $\varpi$ to $S^{\prime}$ :

$$
\begin{aligned}
\Sigma & =d\left(\pi_{A^{\prime}} x^{A^{\prime}}\right) \wedge d\left(\pi_{B^{\prime}} t^{B^{\prime}}+f_{0}\right) \\
& =\pi_{A^{\prime}} \pi_{B^{\prime}} d x^{A^{\prime}} \wedge d t^{B^{\prime}}+\pi_{A^{\prime}} d x^{A^{\prime}} \wedge d f_{0},
\end{aligned}
$$

where we are working over $\mathcal{U}_{0}$. Now

$$
\begin{aligned}
d f_{0} & =d x^{B^{\prime}} \otimes \frac{\partial}{\partial x^{B^{\prime}}} \oint_{\Gamma_{0}} \frac{(\pi . o)^{2}}{(\rho . o)^{2}} \frac{f\left(\rho, \rho_{A^{\prime}} x^{A^{\prime}}\right)}{\pi . \rho} \rho . d \rho \\
& =d x^{B^{\prime}} \oint_{\Gamma_{0}} \frac{\rho_{B}^{\prime}(\pi . o)^{2}}{(\rho . o)^{2}(\pi . \rho)} \frac{\partial f}{\partial P} \rho . d \rho .
\end{aligned}
$$

Where we have used $\frac{\partial}{\partial x^{A^{\prime}}} \rightarrow \rho_{A^{\prime}} \frac{\partial}{\partial P}$. Using this we get 


$$
\begin{aligned}
\Sigma & =\pi_{A^{\prime}} \pi_{B^{\prime}} d x^{A^{\prime}} \wedge d t^{B^{\prime}}+\left(\oint_{\Gamma_{0}} \frac{\pi_{A^{\prime}} \rho_{B^{\prime}}(o . \pi)^{2}}{(o . \pi)^{2}(\pi . \rho)} \frac{\partial f}{\partial P} \rho . d \rho\right) d x^{A^{\prime}} \wedge d x^{B^{\prime}} \\
& =\pi_{A^{\prime}} \pi_{B^{\prime}} d x^{A^{\prime}} \wedge d t^{B^{\prime}}+\frac{1}{2}\left(\oint_{\Gamma_{0}} \frac{(o . \pi)^{2}}{(o . \rho)^{2}} \frac{\partial f}{\partial P} \rho . d \rho\right) d Y \wedge d X \\
& =\pi_{A^{\prime}} \pi_{B^{\prime}} d x^{A^{\prime}} \wedge d t^{B^{\prime}}+(o . \pi)^{2} Q(X, Y) d Y \wedge d X
\end{aligned}
$$

where

$$
Q(X, Y)=\frac{1}{2} \oint_{\Gamma_{0}} \frac{1}{(o . \rho)^{2}} \frac{\partial f}{\partial P} \rho . d \rho .
$$

Putting $o^{A^{\prime}}=(1,0)$, we get the following formula for $\boldsymbol{\Sigma}$ pulled back to $M \times \mathbb{C}^{2}$ :

$$
\Sigma=\pi_{0^{\prime}}^{2}(d T \wedge d X+Q(X, Y) d Y \wedge d X)+\pi_{0^{\prime}} \pi_{1^{\prime}}(d T \wedge d Y-d X \wedge d Z)+\pi_{1^{\prime}}^{2} d Z \wedge d Y .
$$

Calculating $\Sigma$ in the Plebanski formalism from (6.21), (6.22) and (6.23) gives

$$
\begin{aligned}
\Sigma= & \pi_{0^{\prime}}^{2}\left(d T-\Theta_{X X} d Y-\Theta_{T X} d Z\right) \wedge\left(d X+\Theta_{T T} d Z+\Theta_{T X} d Y\right)+ \\
& \pi_{0^{\prime}} \pi_{1^{\prime}}(d T \wedge d Y-d X \wedge d Z)+\pi_{1^{\prime}}^{2} d Z \wedge d Y .
\end{aligned}
$$

Comparing gives the forms $\boldsymbol{\Sigma}^{A^{\prime} B^{\prime}}$ and hence the metric (6.26). The arbitrary function $Q$ corresponds to some arbitrary cohomology element $f$.

\subsection{Example 2. Flat conformal structure.}

Here we show that given a conformal Killing vector for the flat conformal structure, the underlying projective structure is also flat. By the results of [26], we need only consider the conformal Killing vectors $\partial_{T}$ (non-twisting) and $T \partial_{T}+X \partial_{X}$ (twisting), where the flat metric is

$$
g=d T d Y-d X d Z
$$

The non-twisting case is covered by the example of the last section, with $Q(X, Y)=0$, so we know the projective structure is flat and $Z=\mathcal{O}(1)$.

The twisting case is slightly more complicated. One can use the spray picture, but instead we will analyse the twistor space $\mathcal{P} \mathcal{T}$ and show that the space of trajectories of $\mathcal{K}$ is the flat projective structure twistor space $\mathbb{C P}^{2}$ We work on the non-projective twistor space $\mathcal{T}=\mathbb{C}^{4}$ with coordinates $\left(\omega^{A}, \pi_{A^{\prime}}\right)$. The projective twistor space $\mathcal{P} \mathcal{T}$ is a quotient of $\mathcal{T}$ be the Euler homogeneity vector field $\Upsilon=\omega^{A} / \partial \omega^{A}+\pi_{A^{\prime}} / \partial \pi_{A^{\prime}}$. The flat conformal class on the complexified $\mathbb{R}^{2,2}$ and the conformal twisting Killing vector are represented by

$$
g=\varepsilon_{A B} d p^{B} d q^{A}, \quad K=p^{A} \frac{\partial}{\partial p^{A}}
$$


Figure 4: Quotient of the non-projective twistor space by the Euler vector field showing the singular set of $\mathcal{K}$.

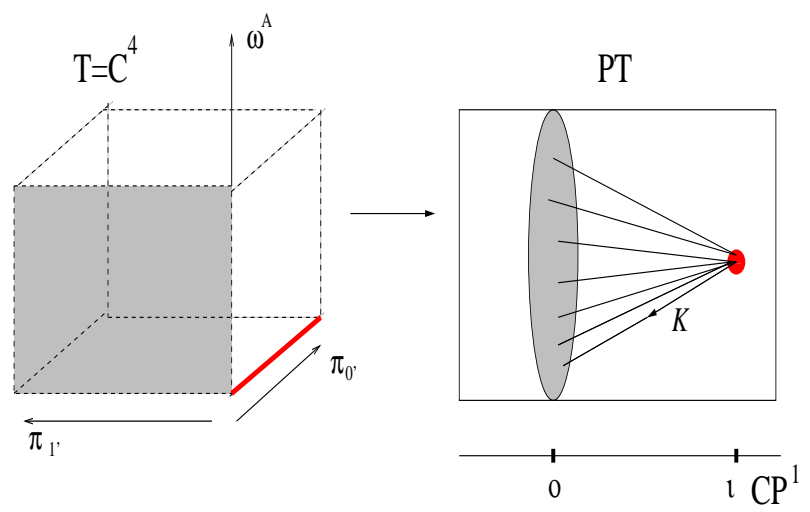

where $x^{A A^{\prime}}:=p^{A} O^{A^{\prime}}+q^{A} \iota^{A^{\prime}}$ are coordinates on $M$. The point $\left(p^{A}, q^{A}\right)$ corresponds to a two-plane in $\mathcal{T}$ given by solutions to the twistor equation $\omega^{A}=x^{A A^{\prime}} \pi_{A^{\prime}}$. The lift of $K$ to $S^{\prime}$ is

$$
\tilde{K}=K+\pi_{1^{\prime}} \frac{\partial}{\partial \pi_{1^{\prime}}},
$$

and the orbits of the induced group action on the non-projective twistor space are

$$
\omega^{A} \rightarrow c \omega^{A}, \quad \pi_{1^{\prime}} \longrightarrow c \pi_{1^{\prime}}, \quad \pi_{0^{\prime}} \longrightarrow \pi_{0^{\prime}}
$$

The holomorphic vector field on $\mathcal{T}$

$$
\mathcal{K}=\omega^{A} \frac{\partial}{\partial \omega^{A}}+\pi_{1^{\prime}} \frac{\partial}{\partial \pi_{1^{\prime}}},
$$

vanishes on the projective twistor space when it is proportional to the Euler vector field. This happens on a set $B=\left\{\left\{\omega^{A}=0, \pi_{1^{\prime}}=0\right\} \cup\left\{\pi_{0^{\prime}}=0\right\}\right\} \subset \mathcal{T}$ which is a union of the line and a hyperplane $\mathbb{C}^{3} \subset \mathcal{T}$. The set $B$ descents to a union of a hypersurface and a point in the projective twistor space (Fig. 4). The minitwistor space $Z$ corresponding to the projective structure $U$ is the factor space of $\mathcal{P} \mathcal{T} / B$ by the trajectories of $\mathcal{K}$. Each trajectory in $\mathcal{T}$ is parametrised by its intersection with the singular surface $\mathbb{C}^{3}$ given by $\pi \cdot o=0$ in $\mathcal{T}$ so the space of trajectories in $\mathcal{P} \mathcal{T}$ is $Z=\mathbb{C P}^{2}$. Two $\mathbb{C P}^{1}$ s in $\mathbb{C P}^{2}$ intersect in a point so the normal bundle of each $\mathbb{C P}^{1}$ is $\mathcal{O}(1)$ and we have a projective structure. To obtain the explicit parametrisation of these $\mathbb{C P}^{1}$ s eliminate $\pi_{0^{\prime}}$ from the twistor equation to get $\pi_{1^{\prime}}=\omega^{A} u_{A}$ where $u_{A}:=$ $p_{A} /\left(p_{B} q^{B}\right)$ parametrise the twistor lines in $Z$ and are coordinates on $U$. The flat metric in $M$ is conformal to (1.3) with $A_{\alpha}, G=z^{2} / 2$ and conformal factor $e^{t}$. 


\section{Outlook}

We have locally classified neutral signature ASD conformal structures with null Killing vectors. Some of these are defined on compact manifolds. It would be interesting to investigate the global properties of other conformal structures we have found.

It would also be interesting to understand in more detail which conformal structures admit special types of metric, for example Ricci-flat or Einstein (in this case the pure Killing vectors must be twist-free [14]). So far the only results we have in this direction are given in Section 6.5. The existence of these special metrics should be related to invariants of the corresponding projective structure.

The recent work of Calderbank [3] extended many of the results obtained in this paper. In particular Calderbank gave a twistor characterisation of ASD conformal structures which admit a geodesic shear free congruence $\iota^{A}$. Not all such congruences give rise to null conformal Killing vectors $K$ such that $\iota^{A} K_{A A^{\prime}}=0$, and Calderbank characterised those which do.

Acknowledgements. We wish to thank Helga Baum, David Calderbank, Claude LeBrun, Lionel Mason, George Sparling and Paul Tod for helpful discussions. S.W. thanks the EPSRC for financial support.

\section{A Appendix}

Here we summarise the required spinor notation and present the calculations leading to a proof of (4.3). We use similar conventions to Penrose and Rindler [22] adapted to neutral signature, but our indices are concrete.

Spin connection and curvature decomposition. As usual, we denote the Levi-Civita connection of the metric by $\nabla$. The 'spin connection coefficients' are defined by

$$
\nabla\left(\mathbf{e}_{C C^{\prime}}\right)=\theta^{D D^{\prime}} \otimes\left(\Gamma_{D D^{\prime} C}^{E} \mathbf{e}_{E C^{\prime}}+\Gamma_{D D^{\prime} C^{\prime}}^{E^{\prime}} \mathbf{e}_{C E^{\prime}}\right),
$$

together with the symmetry requirement $\Gamma_{D D^{\prime} C E}=\Gamma_{D D^{\prime} E C}, \Gamma_{D D^{\prime} C^{\prime} E^{\prime}}=\Gamma_{D D^{\prime} E^{\prime} C^{\prime}}$. These conventions result in the following expressions for differentiation of spinor components, where $\iota^{A}$ is a two-component spinor field over the manifold:

$$
\begin{aligned}
& \nabla_{B B^{\prime}} \iota^{A}=\mathbf{e}_{B B^{\prime}}\left(\iota^{A}\right)+\Gamma_{B B^{\prime} C}{ }^{A} \iota^{C}, \\
& \nabla_{B B^{\prime}} \iota_{A}=\mathbf{e}_{B B^{\prime}}\left(\iota_{A}\right)-\Gamma_{B B^{\prime} A}{ }^{C} \iota_{C},
\end{aligned}
$$

and similarly for a primed spinor field. These are the concrete expressions for the covariant differentiation of spinors using the connections on $S$ and $S^{\prime}$ inherited from the Levi-Civita connection, mentioned in Section 2.1. One can extend the above 
expressions to multi-component objects in the obvious way, allowing covariant differentiation of tensors, which agrees with covariant differentiation using the Levi-Civita connection.

The Riemann tensor has the following spinor decomposition ([22], pg. 236):

$$
\begin{aligned}
R_{a b c d}= & C_{A B C D} \epsilon_{A^{\prime} B^{\prime}} \epsilon_{C^{\prime} D^{\prime}}+\tilde{C}_{A^{\prime} B^{\prime} C^{\prime} D^{\prime}} \epsilon_{A B} \epsilon_{C D} \\
& +\Phi_{A B C^{\prime} D^{\prime}} \epsilon_{A^{\prime} B^{\prime}} \epsilon_{C D}+\Phi_{A^{\prime} B^{\prime} C D} \epsilon_{A B} \epsilon_{C^{\prime} D^{\prime}} \\
& +2 \Lambda\left(\epsilon_{A C} \epsilon_{B C} \epsilon_{A^{\prime} C^{\prime}} \epsilon_{B^{\prime} D^{\prime}}-\epsilon_{A D} \epsilon_{B C} \epsilon_{A^{\prime} D^{\prime}} \epsilon_{B^{\prime} C^{\prime}}\right) .
\end{aligned}
$$

The Weyl spinors $C_{A B C D}, \tilde{C}_{A^{\prime} B^{\prime} C^{\prime} D^{\prime}}$ are completely symmetric, and the traceless Ricci tensor $\Phi_{A B C^{\prime} D^{\prime}}$ is symmetric on each pair of indices. The $C, \tilde{C}$ spinors make up the self-dual and anti-self dual parts of the Weyl tensor. In the language of representation theory, this is the decomposition of $R_{a b c d}$ into irrreducible representations under the action of $S L(2, \mathbb{R}) \times S L(2, \mathbb{R})$ (with $\mathbb{R}$ replaced by $\mathbb{C}$ for the holomorphic case).

Note that in ++-- , spinor components are real. For analytic metrics, we can analytically continue which amounts to allowing the spinors to be complex. The remaining calculations in this appendix are valid in both cases.

Integrability of $\alpha$ and $\beta$ surfaces. We now show that (2.7) and (2.8) are equivalent to the fact that the two-plane distributions defined by $O^{A^{\prime}}$ and $\iota^{A}$ are integrable. The leaves are called $\alpha$-surfaces and $\beta$-surfaces respectively. The argument is well-known in twistor theory. We will do the calculation for the $O^{A^{\prime}}$ case; the $\iota^{A}$ case is identitical.

Let $X=\alpha^{A} O^{A^{\prime}} \mathbf{e}_{A A^{\prime}}, Y=\beta^{A} O^{A^{\prime}} \mathbf{e}_{A A^{\prime}}$ be vector fields, which by definition are in the $\alpha$-planes determined by $o^{A^{\prime}}$. Then if they commute we have:

$$
[X, Y]_{A A^{\prime}}=\left(f \alpha_{A}+g \beta_{A}\right) o_{A^{\prime}},
$$

for some functions $f, g$. Multiplying by $o^{A^{\prime}}$ gives

$$
o^{A^{\prime}}[X, Y]_{A A^{\prime}}=o^{A^{\prime}}\left(X^{B B^{\prime}} \nabla_{B B^{\prime}} Y_{A A^{\prime}}-Y^{B B^{\prime}} \nabla_{B B^{\prime}} X_{A A^{\prime}}\right)=0 .
$$

Substituting the spinor expressions for $X^{A A^{\prime}}$ and $Y^{A A^{\prime}}$ results in

$$
o^{A^{\prime}} O^{B^{\prime}} \nabla_{B B^{\prime}} O_{A^{\prime}}=0
$$

which is (2.8), and it is easy to show this is sufficient as well as necessary.

Twistor distribution and ASD. Locally, the primed spin bundle $S^{\prime}$ is isomorphic to $M \times \mathbb{C}^{2}$. We choose the coordinates on the $\mathbb{C}^{2}$ to be $\pi^{A^{\prime}}$ for $A^{\prime}=0,1$. This vector bundle has a connection inherited from the Levi-Civita connection of 
the metric, and therefore we can find the horizonal lifts $\tilde{\mathbf{e}}_{A A^{\prime}}$ of the $\mathbf{e}_{A A^{\prime}}$, defined by covariantly constant sections. These lifts are as follows:

$$
\tilde{\mathbf{e}}_{A A^{\prime}}=\mathbf{e}_{A A^{\prime}}-\Gamma_{A A^{\prime} B^{\prime}}{C^{\prime}}^{B^{\prime}} \frac{\partial}{\partial \pi^{C^{\prime}}} .
$$

Using the following formula ([22], pg. 247) relating curvature quantities to the derivatives of $\Gamma_{A A^{\prime} C^{\prime}}{ }^{D^{\prime}}$ : and the spinor decomposition of the curvature (A.1) we find

$$
\begin{aligned}
{\left[\pi^{A^{\prime}} \tilde{\mathbf{e}}_{A A^{\prime}}, \pi^{B^{\prime}} \tilde{\mathbf{e}}_{B B^{\prime}}\right]=} & \left(\Gamma_{A A^{\prime} B}{ }^{D}-\Gamma_{B A^{\prime} A}{ }^{D}\right) \pi^{A^{\prime}} \pi^{B^{\prime}} \tilde{\mathbf{e}}_{D B^{\prime}} \\
& +\pi^{A^{\prime}} \pi^{B^{\prime}} \epsilon_{A B} \epsilon^{F^{\prime} Q^{\prime}} \tilde{C}_{A^{\prime} B^{\prime} E^{\prime} Q^{\prime}} \pi^{E^{\prime}} \frac{\partial}{\partial \pi^{F^{\prime}}} .
\end{aligned}
$$

One can see from this that if $\tilde{C}_{A^{\prime} B^{\prime} C^{\prime} D^{\prime}}=0$ then $\pi^{A^{\prime}} \tilde{\mathbf{e}}_{A A^{\prime}}, A=0,1$, forms an integrable distribution. The projection of a leaf of this distribution to $M$ gives an $\alpha$-surface. We have demonstrated that if the metric is anti-self-dual, then given any point $p \in M$ and an $\alpha$-plane at $p$, there is a unique $\alpha$-surface through $p$ tangent to this $\alpha$-plane . This was first shown by Penrose [21], although without using the primed spin bundle. For our purposes the above formulation will be most useful.

Proof of Proposition 1. We have the following identity:

$$
K^{a} R_{a b c d}=\nabla_{b} \nabla_{c} K_{d}-\frac{1}{2}\left(\eta_{, b} g_{c d}-\eta_{, c} g_{b d}+\eta_{, d} g_{b c}\right),
$$

where $\eta$ is the conformal factor appearing in (2.4). Using the curvature decomposition (A.1) to convert this into spinor form, one can calculate

$$
\begin{aligned}
& {\left[K^{A A^{\prime}} \tilde{\mathbf{e}}_{A A^{\prime}}, \pi^{B^{\prime}} \tilde{\mathbf{e}}_{B B^{\prime}}\right]=\left(K^{A A^{\prime}} \Gamma_{A A^{\prime} B}{ }^{D}-\psi_{B}{ }^{D}\right) L_{D}} \\
& -\pi^{B^{\prime}}\left(\phi_{B^{\prime}}^{A^{\prime}} \epsilon_{B}{ }^{A}+\frac{1}{2} \eta \epsilon_{B^{\prime}}^{A^{\prime}} \epsilon_{B}{ }^{A}\right) \tilde{\mathbf{e}}_{A A^{\prime}} \\
& +\pi^{B^{\prime}} \pi^{E^{\prime}}\left(\mathbf{e}_{B B^{\prime}} \phi_{E^{\prime}}^{F^{\prime}}-\Gamma_{B B^{\prime} E^{\prime}}{ }^{G^{\prime}} \phi_{G^{\prime}}^{F^{\prime}}+\Gamma_{B B^{\prime} G^{\prime}}{ }^{F^{\prime}} \phi_{E^{\prime}}^{G^{\prime}}-\frac{1}{4}\left(\mathbf{e}_{B E^{\prime}} \eta\right) \epsilon^{F^{\prime}}{ }_{B^{\prime}}\right) \frac{\partial}{\partial \pi^{F^{\prime}}} .
\end{aligned}
$$

We wish to add a vertical term to $K^{A A^{\prime}} \tilde{\mathbf{e}}_{A A^{\prime}}$ which will cancel all the non- $L_{A}$ terms on the RHS of (A.3). We don't mind about multiples of the Euler vector field since this gets quotiented out on projectivizing. A simple calculation shows that $\tilde{K}$ as defined in (4.4) does the trick.

\section{References}

[1] Barrett, J., Gibbons, G. W., Perry, M. J., Pope, C. N., Ruback, P. J. 1994 Kleinian geometry and the $N=2$ superstring Int. J. Mod. Phys. A. 9, 14571494. 
[2] Bryant, R., Griffiths, P., Hsu, L. Toward a Geometry of Differential Equations, in Geometry, Topology, and Physics, Conf. Proc. Lecture Notes Geom. Topology, edited by S.-T. Yau, vol. IV (1995), pp. 1-76, Internat. Press, Cambridge, MA.

[3] Calderbank, D. 2006 Selfdual 4-manifolds, projective structures, and the Dunajski-West construction math.DG/0606754.

[4] Calderbank, D. 2002 Integrable Background Geometries Preprint.

[5] Dunajski, M. 1999 The twisted photon associated to hyper-Hermitian fourmanifolds. J. Geom. Phys. 30, 266-281.

[6] Dunajski, M. 2002 Anti-self dual four-manifolds with a parallel real spinor. Proc. R. Soc. Lond. A 458, 1205-1222.

[7] Dunajski, M. Mason, L.J., \& Tod, K.P. 2001 Einstein-Weyl geometry, the dKP equation and twistor theory, J. Geom. Phys. 37, 63-92.

[8] Finley, III, J. D., Plebański, J. F. 1979 The classification of all $\mathcal{H}$ spaces admitting a Killing vector, J. Math. Phys. 20 1938-1945.

[9] Fino, A., Pedersen, H., Poon, Y-S., Sorensen, M. W. 2004 Neutral Calabi-Yau structures on Kodaira manifolds. Comm. Math. Phys. 248 255-268.

[10] Graham, C. R. 1987 On Sparling's characterization of Fefferman metrics, Am. J. Math. 109 853-74.

[11] Hitchin, N. J. 1982 Complex manifolds and Einstein's equations. Twistor Geometry and Non-Linear Systems (Lecture Notes in Mathematics vol 970).

[12] Ivanov, S., Zamkovoy, S. 2005 Parahermitian and paraquaternionic manifolds. Differential Geom. Appl. 23 205-234.

[13] Jones, P., Tod, K. P. 1985 Mini twistor spaces and Einstein-Weyl spaces. Class. Quant. Grav. 2, 565-577.

[14] Julia, B., Nicolai, H. 1995 Null-Killing vector reduction and Galilean geometrodynamics. Nuclear Physics B 439 291-323.

[15] Kamada, H. (1999) Neutral hyper-Kahler structures on primary Kodaira surfaces. Tsukuba J. Math. 23, 321-332.

[16] Law, P. 2006 Classification of the Weyl curvature spinors of neutral metrics in four dimensions. To be published in J. Geom. Phys. 
[17] LeBrun, C. 1980 Spaces of Complex Geodesics and Related Structures, D. Phil thesis, Oxford University.

[18] LeBrun, C., Mason, L. J. 2005 Nonlinear Gravitons, Null Geodesics and Holomorphic Discs, math.DG/0504582.

[19] Mason, L. J., Woodhouse, N. M. J. 1996 Integrability, self-duality and twistor theory. LMS Monographs New Series, vol. 15. Oxford University Press.

[20] Nurowski, P., Sparling, G. A. J. 2003 Three-dimensional Cauchy-Riemann structures and second-order ordinary differential equations, Class. Quant. Grav. 20, 4995-5016.

[21] Penrose, R. 1976 Nonlinear gravitons and curved twistor theory. Gen. Relat. Grav. 7, 31-52.

[22] Penrose, R., Rindler, W. 1986 Spinors and space-time, vols. 1 \& 2, Cambridge University Press.

[23] Plebanski, J. F. 1975 Some solutions of complex Einstein equations. J. Math. Phys. 12, 2395-2402.

[24] Sparling, G. A. J., Tod, K. P. 1981 An example of an H-Space. J. Math. Phys. 22 331-332.

[25] Szekeres, P. 1963 Spaces conformal to a class of spaces in general relativity Proc. R. Soc. London A 274, 206-212.

[26] Tafel, J., Wójcik, D. 1998 Null Killing vectors and reductions of the self-duality equations Nonlinearity 11 835-844.

[27] Tafel, J. (1993) Two-dimensional reductions of the self-dual Yang-Mills equations in self-dual spaces. J. Math. Phys. 34, 1892-1907.

[28] Ward, R. S. 1978 A class of self-dual solutions of Einstein's equations Proc. R. Soc. London A 363 289-295.

[29] Ward, R.S. 1985 Integrable and solvable systems and relations among them, Phil. Trans. R. Soc. A315, 451-457.

[30] Ward, R.S. 1990 Einstein-Weyl spaces and $S U(\infty)$ Toda fields, Class. Quantum Grav. 7 L95-L98. 